\title{
Humanized neurofibroma model from induced pluripotent stem cells delineates tumor pathogenesis and developmental origins
}

\author{
Juan Mo, ${ }^{1}$ Corina Anastasaki, ${ }^{2}$ Zhiguo Chen, ${ }^{1}$ Tracey Shipman, ${ }^{1}$ Jason Papke, ${ }^{2}$ Kevin Yin, ${ }^{1}$ David H. Gutmann, ${ }^{2}$ and Lu Q. Le ${ }^{1,3,4}$ \\ 'Department of Dermatology, UT Southwestern Medical Center, Dallas, Texas, USA. ²Department of Neurology, Washington University School of Medicine in St. Louis, St. Louis, Missouri, USA. ${ }^{3}$ Simmons \\ Comprehensive Cancer Center and ${ }^{4}$ Hamon Center for Regenerative Science and Medicine, UT Southwestern Medical Center, Dallas, Texas, USA.
}

\begin{abstract}
Neurofibromatosis type 1 (NF1) is a common tumor predisposition syndrome caused by NF1 gene mutation, in which affected patients develop Schwann cell lineage peripheral nerve sheath tumors (neurofibromas). To investigate human neurofibroma pathogenesis, we differentiated a series of isogenic, patient-specific NF1-mutant human induced pluripotent stem cells (hiPSCs) into Schwannian lineage cells (SLCs). We found that, although WT and heterozygous NF1-mutant hiPSCs-SLCs did not form tumors following mouse sciatic nerve implantation, NF1-null SLCs formed bona fide neurofibromas with high levels of SOX10 expression. To confirm that SOX10+ SLCs contained the cells of origin for neurofibromas, both Nf1 alleles were inactivated in mouse Sox $10^{+}$cells, leading to classic nodular cutaneous and plexiform neurofibroma formation that completely recapitulated their human counterparts. Moreover, we discovered that NF1 loss impaired Schwann cell differentiation by inducing a persistent stem-like state to expand the pool of progenitors required to initiate tumor formation, indicating that, in addition to regulating MAPK-mediated cell growth, NF1 loss also altered Schwann cell differentiation to promote neurofibroma development. Taken together, we established a complementary humanized neurofibroma explant and, to our knowledge, first-in-kind genetically engineered nodular cutaneous neurofibroma mouse models that delineate neurofibroma pathogenesis amenable to future therapeutic target discovery and evaluation.
\end{abstract}

\section{Introduction}

Neurofibromatosis type 1 (NF1), caused by germline mutations in the NF1 gene, is one of the most common human genetic disorders affecting the nervous system. As such, individuals with NF1 are prone to the development of multiple central and peripheral nervous system tumors. Although gliomas predominate in the CNS, children and adults are prone to the formation of 2 major types of peripheral nerve sheath tumors (neurofibromas): (a) plexiform neurofibromas (pNFs) and (b) dermal or cutaneous neurofibromas (cNFs). pNFs typically involve multiple nerves or a nerve plexus, are congenital in origin, disfiguring, associated with abnormal bone growth or erosion, and harbor a lifelong risk of malignant transformation into deadly malignant peripheral nerve sheath tumors (MPNSTs). In contrast, cNFs usually begin to appear in late childhood and early adolescence and continue to increase in size and number throughout life, often numbering in the thousands in some adults.

Previous studies using genetically engineered murine models have revealed that neurofibromas most likely derive from Schwannian lineage cells (SLCs) following somatic loss of the remaining $N f 1$ allele (1-7). Although biallelic Nf1 inactivation in early Schwann cell precursors (SCPs) is an obligate step in murine tumorigenesis, there is also a role for the tumor microenvironment in promoting

Conflict of interest: The authors have declared that no conflict of interest exists. Copyright: () 2021, American Society for Clinical Investigation.

Submitted: May 1, 2020; Accepted: October 21, 2020; Published: January 4, 2021.

Reference information: J Clin Invest. 2021;131(1):e139807.

https://doi.org/10.1172/JCl139807. and facilitating neurofibroma formation and progression (8-11). The interplay between impaired NF1 protein (neurofibromin) inhibition of MEK/ERK-mediated SCP growth and mitogenic signals from non-neoplastic stromal cells has facilitated the identification of novel treatments for plexiform neurofibromas (10, 12-14). Unfortunately, there are no equivalent murine models of discrete $\mathrm{cNF}$ and no accurate humanized in vivo neurofibroma models.

To address this critical barrier, we used a unique series of human induced pluripotent stem cells (hiPSCs) harboring NF1 patient NF1 gene mutations to generate proliferating SCPs and examine the impact of NF1 mutations on Schwann cell lineage differentiation, as well as generate human neurofibromas in mice. Using this approach, we found that NF1 loss delayed SLC differentiation by expanding the pool of progenitors necessary to initiate tumor formation. Moreover, we successfully generated humanized neurofibroma and MPNST models in mice that faithfully phenocopied the analogous tumors in patients. Last, we leveraged this humanized experimental system to identify a subpopulation of SOX10 ${ }^{+}$SCPs, which we exploited to establish what we believe to be the first genetically engineered mouse model of nodular/discrete cNF. Taken together, these humanderived neurofibroma and MPNST models have the potential to serve as tractable platforms for drug identification and screening, as well as to provide unprecedented opportunities to elucidate the mechanisms underlying neurofibroma development and progression.

\section{Results}

Differentiation of isogenic hiPSCs harboring patient NF1 mutations directly into SCPs. SCPs (SCPs) have previously been shown to 
contain the cells of origin for plexiform neurofibroma (4). These progenitors represent intermediate and multipotent stage neural crest-derived cells that emerge following neural tube closure during early embryonic development (15). SCPs provide essential survival signals for developing neurons (16) and guide multipotent cells to specific cell fates and locations (17). In rodents, SCPs are found in the dorsal root ganglion (DRG) (mouse, E12-14; rat, E1415) $(4,18,19)$, where they contribute to the generation of immature Schwann cells (iSCs), which later diversify into nonmyelinating (Remak) and myelinating Schwann cells.

For this reason, we differentiated isogenic hiPSCs (20) directly into SCPs using 50\% DMEM/F12 plus 50\% Neurobasal medium plus N2 and B27, supplemented with SB431542 (activin and a TGF- $\beta$ inhibitor to prevent SMAD signaling, suppress pluripotency, and prevent mesoderm/endoderm induction), a GSK3 inhibitor (CHIR99021, to activate WNT signaling), and higher concentrations of neuregulin $\beta-1$ to support glial fate differentiation (21). Before differentiation, the cells formed colonies (Figure 1A and Supplemental Figure 1A; supplemental material available online with this article; https://doi.org/10.1172/JCI139807DS1), which are characteristic of hiPSCs, and were immunopositive for pluripotent markers, including NANOG, SOX2, Oct3/4, and TRA1-60 (Figure 1A). The pluripotency of these hiPSCs was also confirmed by teratoma formation (Supplemental Figure 1B). Following differentiation, the typical SCP cell shape (elongated bipolar or tripolar dendrites) was distinct from that of hiPSCs (Figure 1). The successful differentiation of hiPSCs into SCPs was confirmed by decreased Oct3/4 and NANOG expression (Figure 1B and Supplemental Figure 2, A and B) and increased expression of SCP markers, including SOX10, HOXb7, GAP43, AP2a (Figure 1, B and $\mathrm{C}$ ), and p75 protein (Figure $1 \mathrm{~B}$ and Figure 2, $\mathrm{A}-\mathrm{C}$ ) as well as $\mathrm{MPZ}$, CDH19, ITGA4, and PLP mRNA (Figure 2D). We found that the adult neural stem cell marker nestin was also expressed in these hiPSC-derived SCPs (hiPSC-SCPs) (Figure 1C). SOX10 and AP2a contribute to the gene regulatory network required for neural crest formation during the emigration of neural crest progenitors from the neural tube through an epithelial-mesenchymal transition (EMT). The colocalization of SOX10 and HOXb7 in hiPSC-SCPs (Figure 1B) suggests that the SOX10-expressing cell population also contained HOXb7-expressing cells, which we previously found to contain the cells of origin for pNFs and cNFs in mice (6).

To determine whether these cells were proliferating, we incubated the cells with BrdU and then costained them for the SCP markers AP2a, SOX10, and HOXb7 (Supplemental Figure 1C). We observed colocalization of these SCP markers with BrdU, indicating that these cells were proliferating, with quantification showing that $\mathrm{NF1}^{-/-}$hiPSC-SCPs had the highest numbers of proliferating cells (Supplemental Figure 1, D-F).

NF1 loss impairs Schwann cell lineage differentiation. Since neurofibromin functions as a negative growth regulator through its ability to accelerate the conversion of active GTP-bound RAS to inactive GDPbound RAS, loss of NF1 results in RAS pathway activation, leading to aberrant growth of SLCs and the formation of neurofibromas (22, 23). In addition, neurofibromin also regulates the differentiation and proliferation of several nervous system cell types, including neural stem cells and neuroglial progenitors in vitro and in vivo in a cell type-, cell function-, and brain region-specific manner $(24,25)$.
To evaluate the role of the NF1 gene in SLC differentiation, we examined isogenic $\mathrm{NF1}^{+/+}$(WT isogenic control), $\mathrm{NF}^{+/-}$(heterozygous patient-based $\mathrm{NF1}$ mutation), and $\mathrm{NF}^{-{ }^{--}}$(homozygous patient-based NF1 mutation) hiPSCs. First, following differentiation, we observed decreased expression of the Oct3/4 and NANOG pluripotent markers (Supplemental Figure 2, A and B). In addition, the percentage of p75-expressing cells (SCPs) among the isogenic lines decreased from $90.4 \%$ in $\mathrm{NF}^{+/+}$hiPSC-SCPs to $81.1 \%$ and $62.5 \%$ in $\mathrm{NF}^{+/-}$and $\mathrm{NF}^{-/-}$hiPSC-SCPs, respectively (Figure 2, $\mathrm{A}-\mathrm{C}$ ). Second, we found that $\mathrm{NF}^{-/-}$hiPSC-SCPs had lower (2- to 8-fold change) mRNA levels of SLC markers, including $M P Z$, CDH19, PLP, SOX1O, and ITGA4A (Figure 2D). Third, differentiated $\mathrm{NF}^{-/-}$hiPSC-SCPs expressed higher mRNA levels (2- to 10-fold change) of NCSC markers (TWIST, SLUG, and SNAIL) (Supplemental Figure 2, C-E) relative to their control $\mathrm{NF}^{+/+}$counterparts. Fourth, when we evaluated hiPSC-SCPs for the percentage or frequency of cells that could form self-renewing and multipotent neurospheres (26), $\mathrm{NF}^{-/-}$hiPSC-SCPs neurospheres had $11.84 \%$ larger average diameters, $58.82 \%$ greater numbers of neurospheres per well, and $84.61 \%$ higher frequencies of neurosphere formation relative to $\mathrm{NF}^{+/+}$hiPSC-SCPs (Figure 2, E-G). Taken together, these findings suggest that NF1 loss increased the number of SLC stem cells (SCPs) (19).

Next, we evaluated the effect of NF1 loss on SCP proliferation. While $\mathrm{NF}^{-/-}$hiPSC-SCPs had increased RAS activity at baseline relative to $\mathrm{NF}^{+/+}$controls (Supplemental Figure 2F), the addition of EGF further increased RAS-GFP levels in $\mathrm{NF}^{+/+}$and $\mathrm{NF}^{+/-}$hiPSC-SCPs, but not in $\mathrm{NF}^{-/-}$hiPSC-SCPs (Supplemental Figure 2F). This result demonstrates that NF1 loss in SCPs results in maximal RAS activation. As a result, cell proliferation was maximally increased in $\mathrm{NF}^{-/-}$hiPSC-SCPs (Supplemental Figure $2 G)$. Western immunoblotting revealed increased expression of GAP43 (Figure 2H and Supplemental Table 1), an essential marker for the cells of origin in neurofibroma, and slightly decreased expression of p53 (Figure $2 \mathrm{H}$ and Supplemental Table 1), which is important for regulating EMT and stemness (27). Interestingly, we consistently observed increased expression of total ERK (t-ERK) in $\mathrm{NF}^{-/-}$hiPSC-SCPs (Figure $2 \mathrm{H}$ ). This increase in newly synthesized ERK might additionally influence tumor formation by elevating the pool of activatable ERK (28). Collectively, these observations establish that NF1 loss delayed SLC differentiation, with $\mathrm{NF}^{+/-}$and $\mathrm{NF1}^{-/-}$cells maintaining a more stem cell-like state, thus expanding the population of cells with the capacity to serve as the cells of origin for neurofibroma (4).

Next, we examined the impact of $\mathrm{Nf1}$ inactivation on mouse embryonic DRG/nerve root neurosphere cells (DNSCs), which contain the cells of origin for plexiform neurofibroma (4) (Supplemental Figure 2, H and I). Adeno-Cre virus-infected $\mathrm{Nf}^{f l / f l}$ DNSCs, in which $N f 1$ loss was confirmed at the DNA (Supplemental Figure 2J) and protein (Figure 2I) levels, had higher protein expression of the adult neural stem cell marker nestin (Figure 2I) and higher mRNA levels of other stem cell markers (Ednrb, Lgr5, Sox2, Ccnd2, Cd133, Igf2bp2, Lif, Olfm4, and Hopx) (Figure 2J), while concomitantly expressing lower mRNA levels of Schwann cell markers (MPZ, ErbB3, p75, Ngfr, Sox10, Dhh, S100 $\beta$, and Krox2O) (Figure 2J). We also observed that adeno-Cre virus-infected $N f f^{f l / f l}$ DNSCs formed neurospheres, whereas GFP virus-infected $N f 1^{f l / f l}$ DNSCs 
A
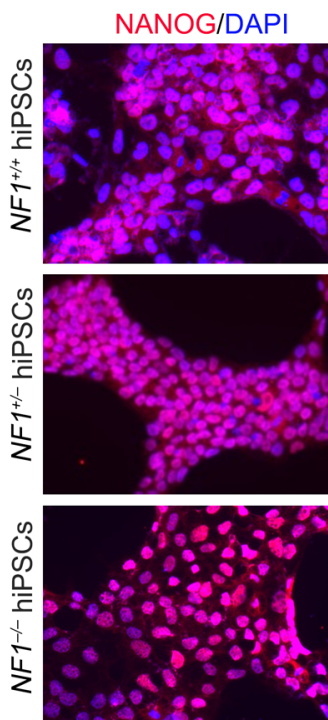

B
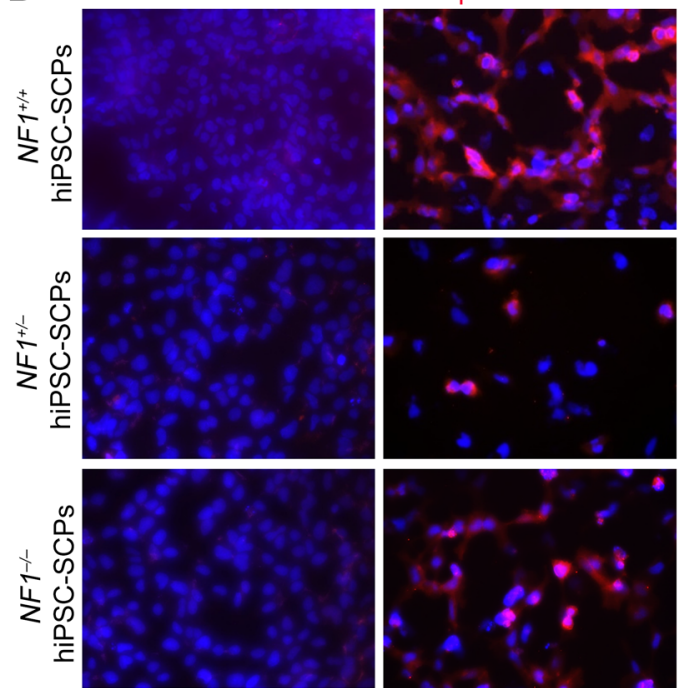

C
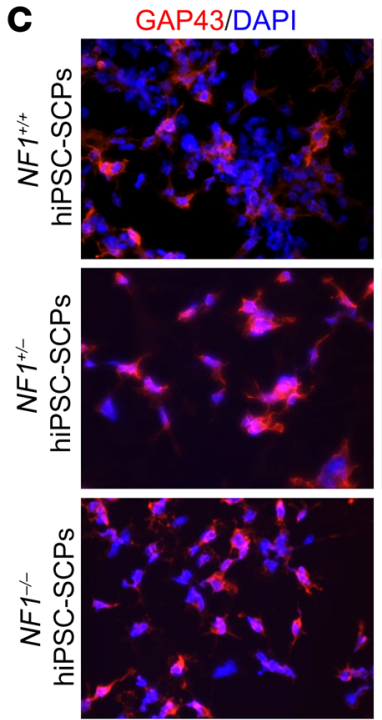

SOX2/DAPI
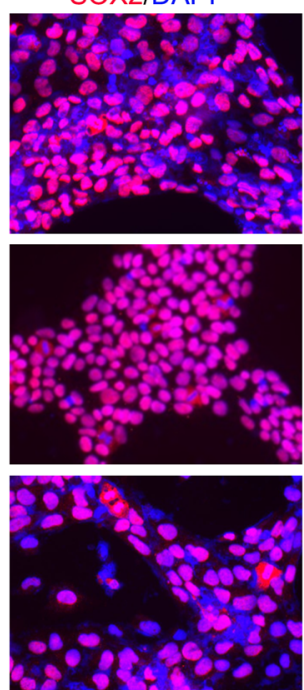

p75/DAPI
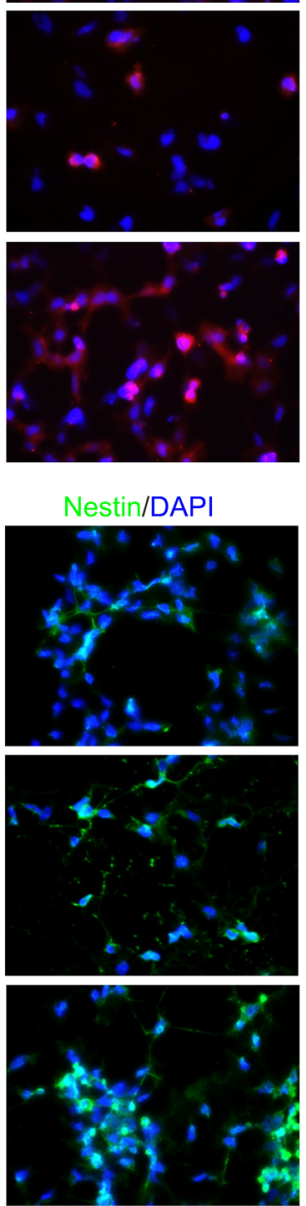

Oct3/4/DAPI
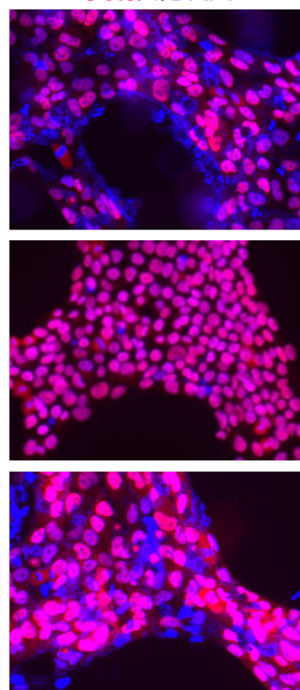

SOX10
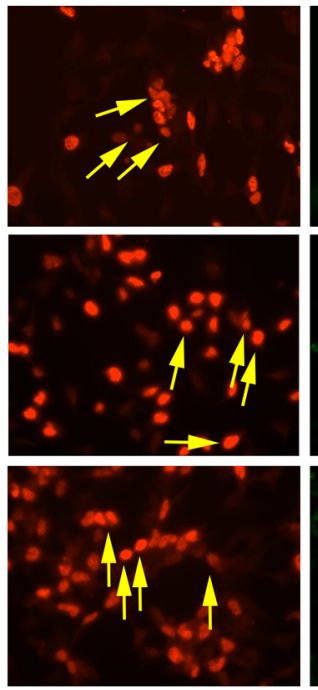

SOX10/DAPI
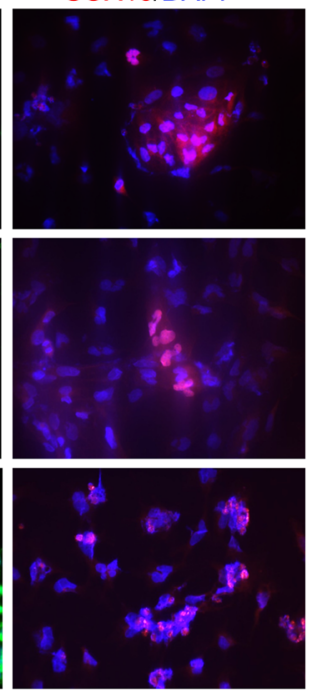

TRA1-60/DAPI
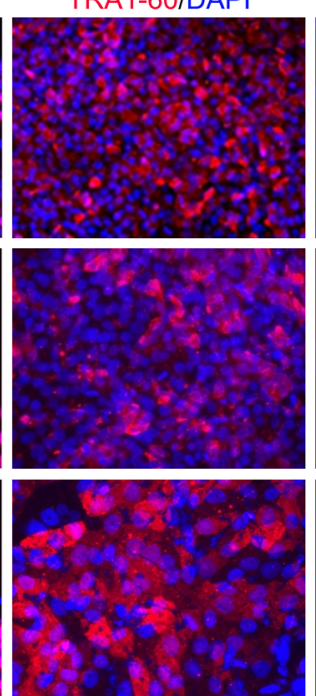

$\mathrm{HOXb7}$
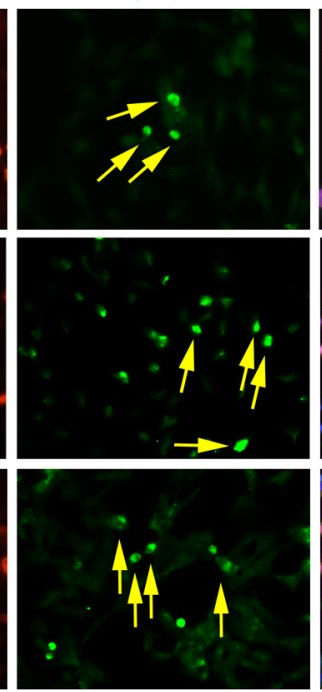

AP2a/DAPI
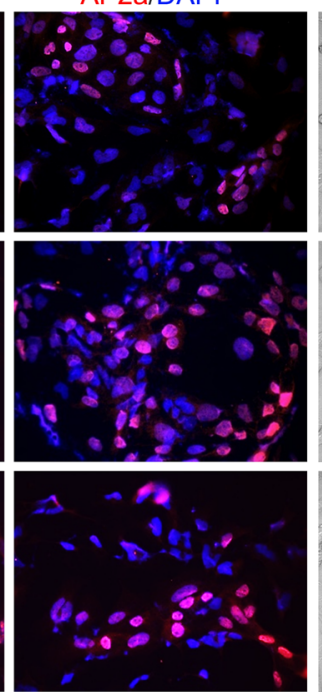

p75/DAPI

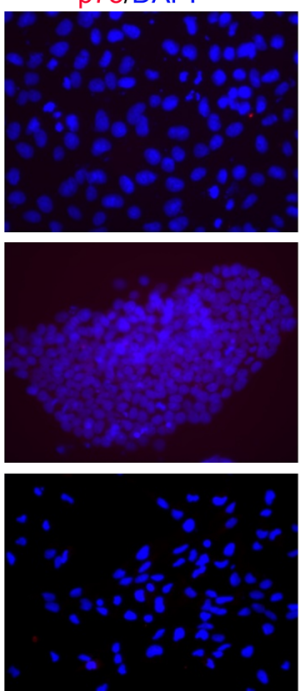

SOX10/HOXb7/DAPI

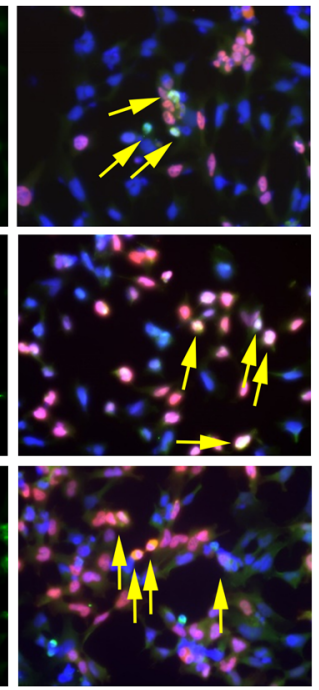

BF

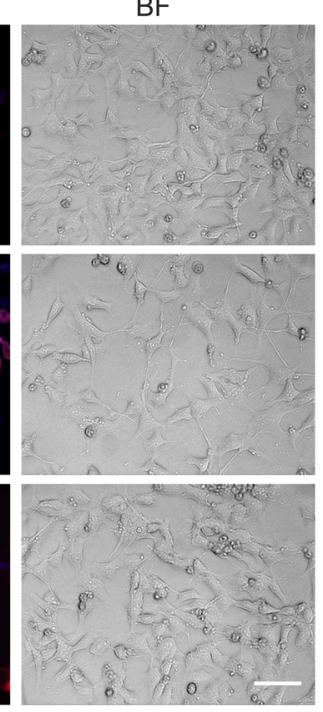


Figure 1. Differentiation of hiPSCs to SCPs. (A) Pluripotency of isogenic $\mathrm{NFT}^{+/+}, \mathrm{NF}^{+/-}$, and $\mathrm{NF}^{-1^{--}}$hiPSCs was confirmed by expression of NANOG, Oct3/4, SOX2, and TRA1-60. (B) Differentiation of isogenic hiPSCs into SCPs was confirmed by negative staining for Oct $3 / 4$ on day 6 and positive staining for $\mathrm{p75}, \mathrm{SOX} 10$, and HOXb7 on day 10 . Yellow arrows depict the colocalization of SOX10 and HOXb7 in hiPSC-SCPs. (C) Differentiation of isogenic hiPSCs into SCPs was confirmed by positive staining for GAP43 and nestin on day 10 and for SOX10 and AP2a on day 20, respectively. The morphology was distinct from that of original hiPSCs 10 days after differentiation. BF, bright-field. Scale bar: $50 \mu \mathrm{m}$.

did not form neurospheres following adenovirus infection (Supplemental Figure 2K). Similarly, $N f 1$ loss resulted in higher RAS activity (Supplemental Figure 2L) and cell proliferation (Supplemental Figure 2M) relative to the adeno-GFP virus-infected $N \mathrm{fl}^{\mathrm{flfl} l}$ DNSC controls. Mirroring our findings in the human SCPs, the addition of EGF further increased RAS activity in $\mathrm{Nf}^{1 / / \mathrm{l}}$-GFP virus-infected, but not $N f^{1 / f / l}$ adeno-Cre virus-infected, DNSCs (Supplemental Figure 2L). In some neural lineages, NF1 loss increases PI3K signaling in addition to MAPK signaling (29). However, we did not observe elevated phosphorylated-S6 (p-S6) kinase levels in $\mathrm{Nf1}^{-1-}$ DNSCs (Figure 2I). The higher levels of GAP43 protein (Figure $2 \mathrm{I})$ and mRNA (Supplemental Figure $2 \mathrm{~N})$ ( $\sim 15$ fold change) in Cre virus-infected $N f^{f / / l}$ DNSCs were consistent with levels detected in human $\mathrm{NF1}^{-/}$iPSCs-SCPs (Figure $2 \mathrm{H}$ ). These results reveal a precise coordination between proliferation and differentiation in SLCs relevant to neurofibroma development.

hiPSCs with patient NF1 mutations generate humanized neurofibromas in mice. During the differentiation of hiPSCs into migrating neural crest cells and SCPs, we discovered that the homeobox protein HOXb7 colocalized with SOX10 expression (Figure 1). Since we have previously shown that HOXb7 serves as a marker to trace the cells of origin for neurofibromas in mice (6), we hypothesized that these differentiated hiPSC-SCPs contain the tumor-initiating cells that could potentially give rise to neurofibromas within a permissive microenvironment. To test this hypothesis, we implanted SOX10/HOXb7-expressing $\mathrm{NF}^{-1 /}$ hiPSC-SCPs into the sciatic nerves of immunodeficient athymic $(n u / n u)$ mice. These engrafted $\mathrm{NF1}^{-1-}$ hiPSC-SCPs survived, proliferated, and formed bona fide masses (plexiform neurofibromas) that completely recapitulated all the histological features of human plexiform neurofibromas, including hypercellularity, disorganization of the nerve tissue architecture, and Schwann cells with wavy nuclei (Figure 3, A and B). The neoplastic cells in these neurofibromas were immunopositive for the human-specific Ku80 marker, and all tumors contained $\mathrm{S} 00 \beta^{+}, \mathrm{GAP}^{+} 3^{+}, \mathrm{SOX}_{10}{ }^{+}, \mathrm{HOXb7}^{+}$, and $\mathrm{Iba}^{+}$(macrophage marker) cells (Figure 3A, right sciatic nerve) with colocalization of Ku80 and GAP43, and had increased levels of p-ERK and p-s6 when compared with the control (Figure 3A, left sciatic nerve). These observations suggest that the neoplastic cells originated from the implanted $\mathrm{NF}^{-1-}$ hiPSC-SCPs that differentiated into GAP $43^{+}$SLCs within a favorable nerve microenvironment. In contrast, when SOX10/HOXb7-expressing $\mathrm{NF}^{+/-}$hiPSC-SCPs were implanted into the left sciatic nerve, the human-derived $\mathrm{Ku} 80^{+}$ cells survived and differentiated into SLCs but did not generate neurofibromas (Figure 3A), confirming that $N F 1$ loss is a requisite step for neurofibroma development. Interestingly, expression of the adult neural stem cell marker nestin, in addition to HOXb7, was seen in these human pNF lesions (Figure 3B), which mirrors the expression of nestin, HOXb7, and SOX10 in hiPSC-SCPs (Figure 1) and in E13.5 DNSCs (Figure 2).

The nerve microenvironment promotes neural crest stem cell differentiation into SLCs and the formation of neurofibromas. Previous studies have revealed that the local nerve microenvironment is important for neurofibroma formation (30). To examine this requirement, we differentiated hiPSCs into neural crest stem cells (NCSCs), a precursor stage of SLCs, as confirmed by decreased expression of the Oct3/4 pluripotency marker and increased expression of the NCSC markers p75 and HNK1 (Figure $4, \mathrm{~A}-\mathrm{C})$. Although activation of the bone morphogenetic pathway (BMP) suppressed the specification of NCSCs (31), addition of LDN193189, a potent BMP inhibitor, did not increase the expression of NCSC markers (e.g., p75), as previously reported in mice (32).These differences might reflect endogenous BMP expression in hiPSC lines (33) or the need for BMP during a critical developmental window in mice (34).

Importantly, when $\mathrm{NF}^{-/}$hiPSC-derived NCSCs (hiPSCNCSCs) were subcutaneously implanted into $n u / n u$ mice, we only observed the formation of cartilage (Figure 4D and Supplemental Figure 3A), another neural crest cell-derived tissue. This cartilage was positive for p-ERK, indicating activation of MEK signaling and NF1 loss (Supplemental Figure 3A). In striking contrast, $\mathrm{NF1}^{-/-}$ hiPSC-NCSCs implanted into the sciatic nerve generated both cartilage and cNFs (Figure 4E and Supplemental Figure 3). However, $\mathrm{NF1}^{+/+}$hiPSC-NCSCs subcutaneously implanted or injected into the left sciatic nerve of the same mice did not generate neurofibromas (Figure 4E). To determine whether there might be other cells present in the microenvironment that provided critical support for cNF development, we immunostained for fibroblast, endothelial cell, macrophage, and mast cell markers, but observed no obvious differences between subcutaneously and sciatic nerve-implanted hiPSC-NSCSs (Supplemental Figure 3B). These findings confirm that, while hiPSC-NCSCs can give rise to different neural crest derivatives, including chondrocytes and SLCs in vivo, consistent with previous work (35), the local nerve microenvironment is essential for the differentiation of $\mathrm{NF1}^{-1-} \mathrm{NCSC}$ into SLCs and the formation of neurofibromas.

Benign human-derived neurofibroma transformation into MPNSTs. Neurofibroma progression to MPNSTs in patients with NF1 occurs with a frequency of $8 \%-15 \%$ and is associated with additional genetic changes, including amplification and overexpression of oncogenic receptor tyrosine kinases (EGFR, PDGFR, $M E T$ ) or growth factors (neuregulin-1, hepatocyte growth factor), coupled with a loss of tumor suppressor genes (CDKN2A, PTEN, TP53, or components of the polycomb-repressive complex 2 [PRC2]) (36-47). To model genetic cooperativity between NF1 loss and one of the other molecular events reported in human MPNSTs, we used CRISPR/Cas9 to mutate TP53 in NF1 ${ }^{-1-}$ hiPSCs. We confirmed the loss of NF1 and P53 expression in $\mathrm{NF1}^{-1-}$ hiPSCs by Western blotting (Figure 5, A and B, Supplemental Figure 4, A and B) and quantitative PCR (qPCR) (Figure 5C and Supplemental Figure 4C). As predicted, mRNA expression of the TP53 target gene $p 21$ was likewise decreased (Figure 5C and Supplemental Figure 4D). 
A

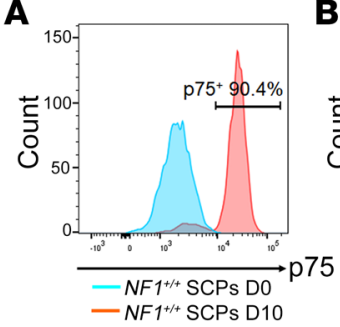

B

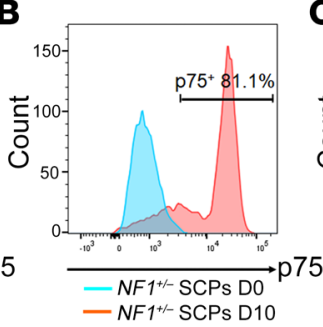

C

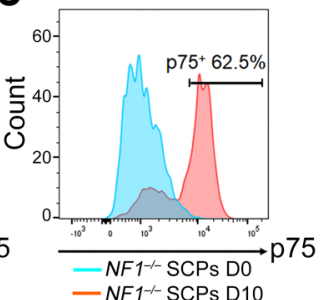

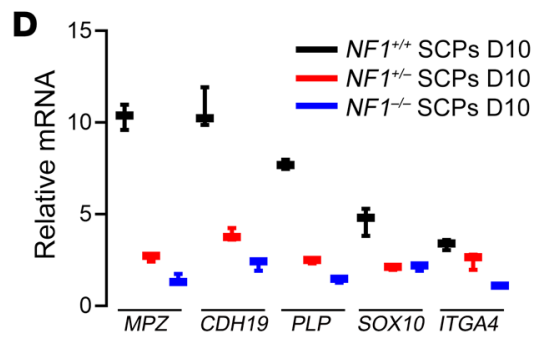

E

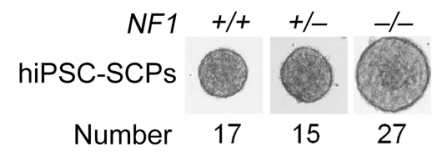

Ave. diameter 98.56100 .85110 .23

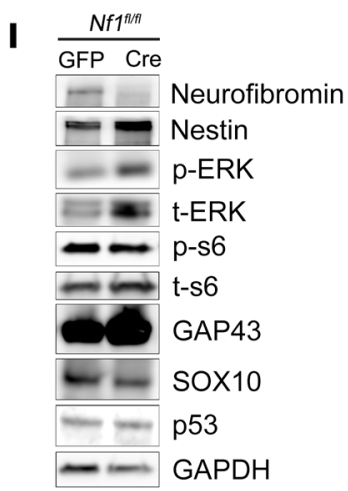

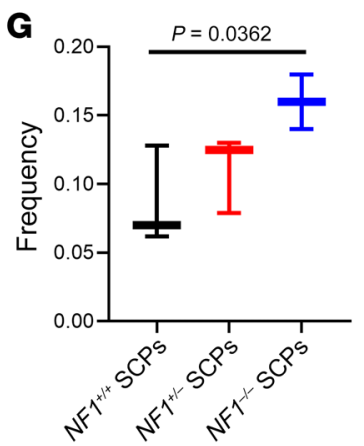

$\mathbf{H}_{\text {hiPSC-SCPs }}$
NF1 $\overline{+/-\quad-1-}$

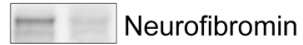

p-ERK

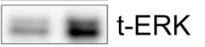

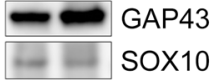

- p53
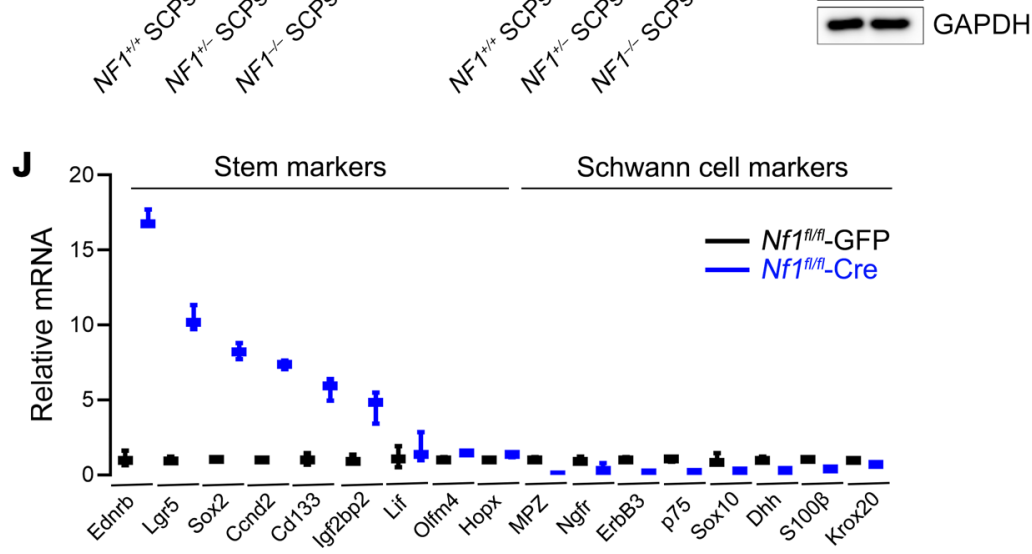

Figure 2. NF1 loss impairs Schwann cell differentiation by maintaining stemness. (A-C) Flow cytometry was performed to measure the percentage of

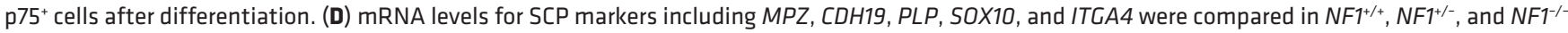
hiPSC-SCPs. (E-G) hiPSCs were grown in SCP-DM for 4 days, followed by suspension culture for an extra 6-14 days. Neurosphere numbers were counted (E), and the average (Ave.) diameters were calculated (F) ( $n=15-27 /$ group). Comparisons among groups were performed by 1-way ANOVA. (C) Frequencies of isogenic hiPSC-SCPs were assessed as the percentage of cells that formed neurospheres. Comparisons among groups were performed by 1-way ANOVA.

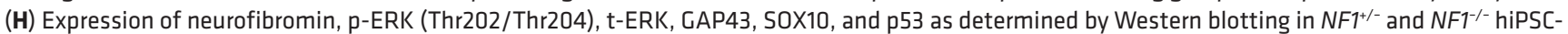
SCPs, with GAPDH used as an internal loading control. (I) Protein expression of neurofibromin, nestin, p-ERK, t-ERK, p-S6 (Ser240/244), t-S6, GAP43, SOX10, and $\mathrm{p} 53$ was measured by Western blotting in adeno-GFP virus-infected and adeno-Cre virus-infected E13.5 Nff $7^{f / f l}$ DNSCs. (J) mRNA levels of stem cell markers (Ednrb, Lgr5, Sox2, Ccnd2, Cd133, Igf2bp2, Lif, Olfm4, and Hopx) and Schwann cell markers (MPZ, Ngfr, ErbB3, p75, Sox10, Dhh, S100 3 , and Krox20) were compared between GFP adenovirus-infected and Cre adenovirus-infected E13.5 Nf $7^{f / f l}$ DNSCs. For the box-and-whisker plots in D, F, G and J, the plots show the median (line) and lower and upper quartiles (box), and the ends of the whiskers represent the lowest and highest values. Comparisons among groups were performed by 1-way ANOVA. DO, day 0; D10, day 10.

The pluripotency of $\mathrm{NF}^{-/-}$single guide TP53 (sgTP53) hiPSCs was established by positive staining for NANOG, SOX2, and Oct3/4 (Figure 5D) and their ability to form teratomas (Supplemental Figure $4 \mathrm{E}) . \mathrm{NF}^{-1-}$ sgTP53 hiPSCs were differentiated into SCPs, which was confirmed by expression of AP2a, p75, GAP43, SOX10, and nestin proteins (Figure 5D) and increased mRNA levels of HOXb7, SOX10, MPZ, CDH19, ITGA4, ErbB3, p75, and GAP43 (Figure 5E). Although $\mathrm{NF}^{-/-}$sgTP53 hiPSC-SCP proliferation was higher than that observed in $\mathrm{NF}^{-/}$single guide scrambled ( $\mathrm{SgSCr}$ ) hiPSC-SCPs (Figure 5F), the expression of SCP markers (SOX10, $M P Z, C D H 19, p 75, I T G A 4$, and ERBB3) was lower (Figure 5G). In contrast, the expression of stem markers (SOX2, SLUG, SNAIL, and TWIST) was higher in $\mathrm{NF1}^{-/-} \mathrm{sgTP53}$ hiPSC-SCPs relative to $\mathrm{NF1}^{-/}$sgScr hiPSC-SCPs (Figure 5H). Consistent with prior mouse modeling experiments, implantation of $\mathrm{SOX} \mathrm{O}^{+} \mathrm{NF1}^{-/-} \mathrm{sgTP} 53$ hiPSC-SCPs generated human MPNSTs similar to their human

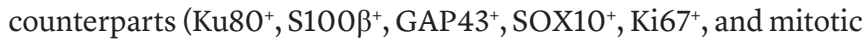
marker p-histone- $3^{+}$cells) (Figure 6). These findings suggest that maintenance of a stem cell or earlier Schwann cell lineage stage probably contributes to malignant transformation (48).

SOX10-expressing cells contain neurofibroma-initiating cells. Because SOX10 is highly expressed in both neurofibromas and MPNSTs (Figures 3 and 6), we hypothesized that SOX10-expressing cells contain the tumor-initiating cells that give rise to neurofibromas. This hypothesis is supported by our observation of SOX10 and HOXb7 colocalization in hiPSC-SCPs (Figure 1) and the finding that HOXb7 serves as a marker to trace the cells of origin for neurofibroma (6). To test this hypothesis, we depleted $N f 1$ 


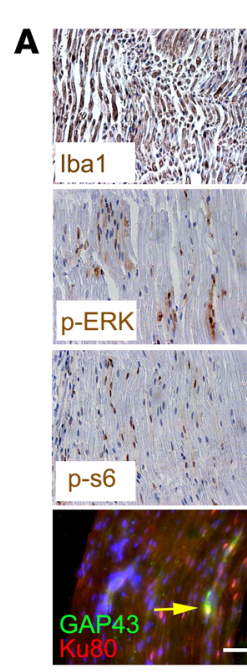

B

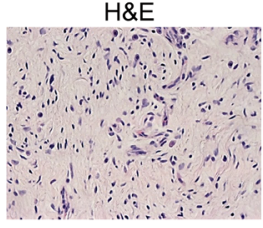

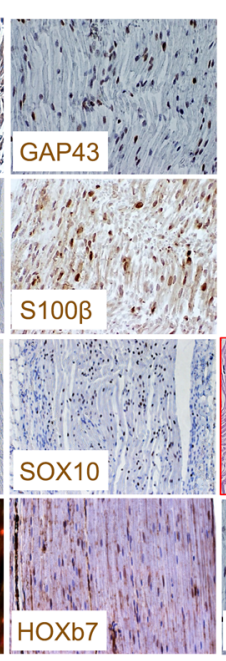

GAP43

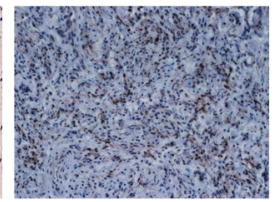

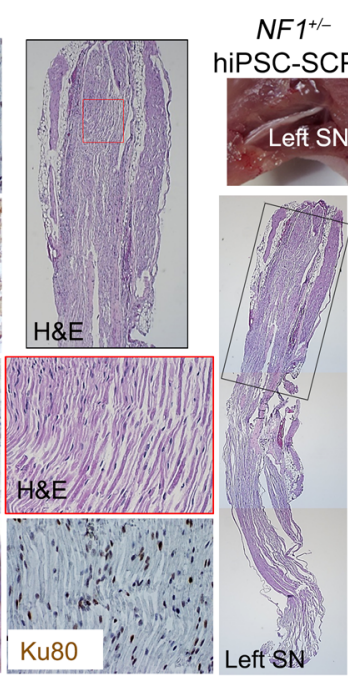

S100ß

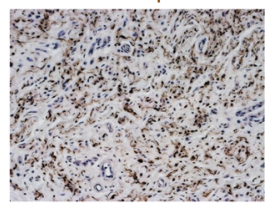

$N F 1^{-/-}$ hiPSC-SCPS
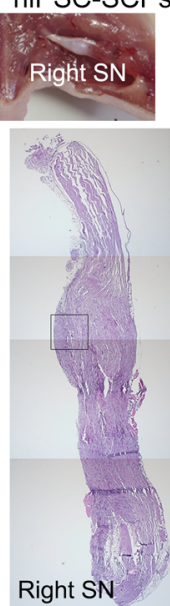

SOX10

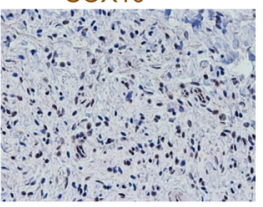

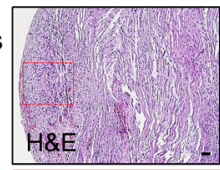

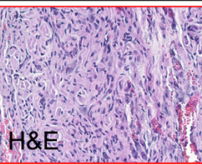

हि:
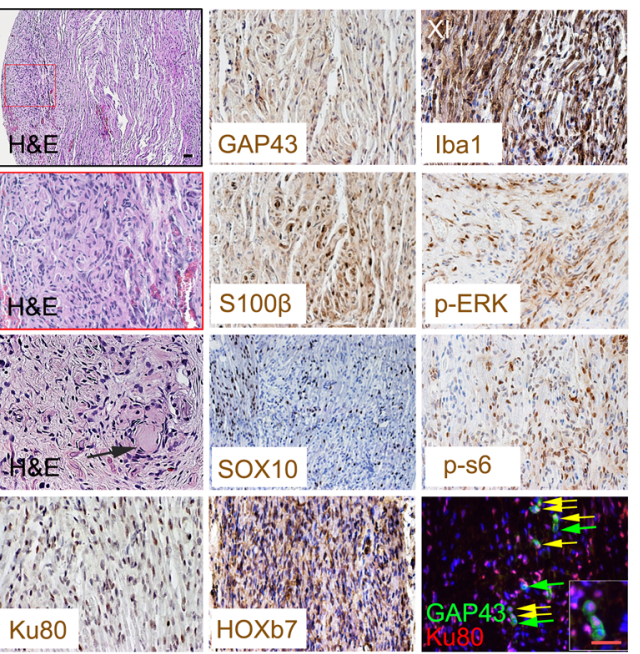

$\mathrm{HOXb7}$

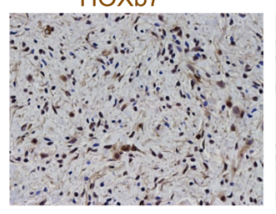

Nestin

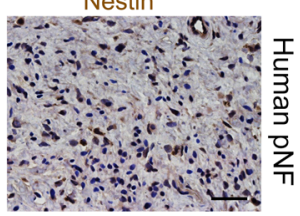

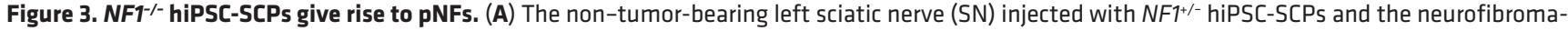
bearing right sciatic nerve injected with $\mathrm{NF}^{-/-}$hiPSC-SCPs were fully characterized by $\mathrm{H} \& \mathrm{E}$ staining as well as immunostaining with the human-specific Ku80, GAP43, S100 $\beta$, SOX10, HOXb7, p-ERK, p-s6, and Iba1 antibodies. Yellow arrows show the colocalization of GAP43+ and Ku80+ cells. Green arrows show GAP43+Ku80- cells. Black arrow shows the Meissner-like corpuscle in the neurofibroma. $n=5$. (B) Characterization of human pNF tissue by H\&E staining and immunostaining for GAP43, S100ß, SOX10, HOXb7, and nestin. SN, sciatic nerve. Scale bars: $50 \mu \mathrm{m}$ and $25 \mu \mathrm{m}$ (inset in A).

expression in SOX10 lineage-derived cells in mice by conditional transgenesis. Since $N f 1$ inactivation in noninducible (constitutive) Sox10-Cre mice results in neonatal lethality, we crossed tamoxifen-inducible Sox1O-CreERT2 mice with $N f 1^{f / f l}$ mice to generate Sox10-CreERT2 $\mathrm{Nf}^{1 / \mathrm{ll} l}$ mice. Following tamoxifen treatment, LacZ was expressed in the spinal cord (Supplemental Figure 5A) and sciatic nerve (Supplemental Figure 5B). We also confirmed tamoxifen-induced $N f 1$ recombination (49) (Supplemental Figure 5C). By 6 months after tamoxifen exposure $(9,50)$, these mice began to show clinical signs characteristic of pNF development (scruffy fur, hunched back, limping, limb paralysis) (Supplemental Figure 5, D and E) and had lower average body weights and worse survival compared with control mice (Supplemental Figure 5, F and G). We also consistently observed that mice in the $\mathrm{Nf}^{f t /-}$ group died from tumor development much faster than did those in the $\mathrm{Nf1}^{\mathrm{fl} / \mathrm{fl}}$ group (Supplemental Figure 5G). Spinal cord dissection revealed enlarged DRGs at the cervical, thoracic, and lumbar levels (Figure 7A). We also observed classic giant, diffuse pNFs within the left hind limb, causing left leg gigantism in some Sox10-CreERT2 $\mathrm{Nf1}^{\mathrm{fl} / \mathrm{fl}}$ mice (Figure 7B). Histological and molecular analysis of these enlarged DRGs and legs revealed tumors that were immunopositive for S100 $\beta$, GAP43, and SOX10 expression, as well as mast cell infiltration (Figure 7), similar to human pNFs (Figure 3B). We confirmed $N f 1$ loss in these tumors by genotyping and Western blotting (Supplemental Figure 5, H and I).

Next, we examined the role of NF1 in Schwann cell differentiation in vivo. In contrast to control mice, tamoxifen-treated Sox1O-CreERT2 Nf1-mutant ( $N f^{f l / f l}$ or $\left.N f 1^{f l /-}\right)$ mice had more com- pact nerve bundles with fascicular tissue architecture (Supplemental Figure 6A). Longitudinal sections of the sciatic nerves showed hypercellular, but well-organized, tissue architecture in the Nf1-mutant mice (Supplemental Figure 6A). The sciatic nerves of Nf1-mutant mice had multiple axons (TUBB3-stained axons) without myelin (MPZ-stained myelin) (Supplemental Figure 6B, middle and bottom panels), representing an undifferentiated stage. Transmission electron microscopic (TEM) analysis further revealed that the sciatic nerves of normal mice had predominantly myelinated axons with 1 surrounding Schwann cell. However, the sciatic nerves of $\mathrm{Nf1}$-mutant mice contained many unmyelinated and immature axons (Supplemental Figure 6C, bottom left panel). Additionally, we observed increased numbers of cells between myelinated axons in the $\mathrm{Nf1}$-mutant nerves relative to controls (Supplemental Figure 6C, bottom right panel). These in vivo observations in $\mathrm{Nf1}$-mutant mice are consistent with our in vitro observation (Figure 2 and Supplemental Figure 2) that Nf1 loss impaired Schwann cell differentiation during development.

Temporal $\mathrm{Nf1}$ deletion in $\mathrm{SOX} 1 \mathrm{O}^{+}$cells generates nodular/discrete cNFs. In addition to developing plexiform neurofibroma, Sox10CreERT2 $N f 1^{f / f l}$ mice also developed multiple discrete skin papules on their backs and necks (Figure 8A and Supplemental Figure 7). To determine whether these lesions were restricted to the dermis, we carefully dissected the skin to demonstrate that all tumors were exclusively confined to the dermis, as seen in human cNFs (Supplemental Figure 7B). In this respect, Sox10-CreERT2 $\mathrm{Nf1}^{f / f / l}$ mice recapitulated the characteristic body location, skin thickness, and cutaneous restriction of these tumors in their human counterparts. 

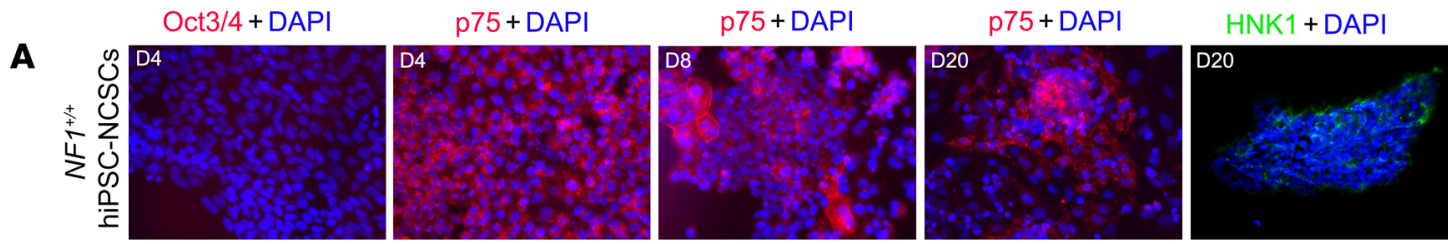

B
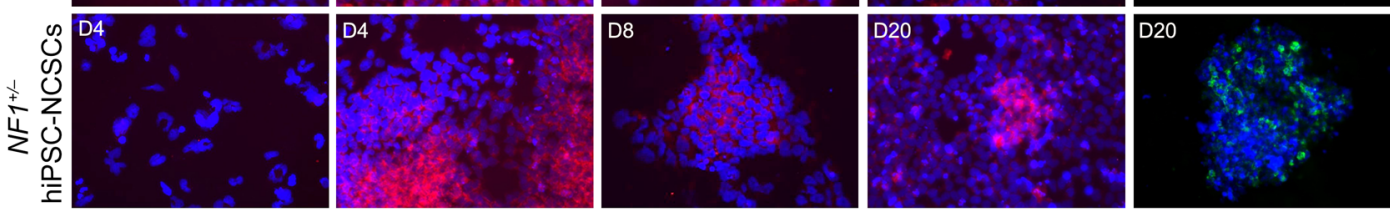

C
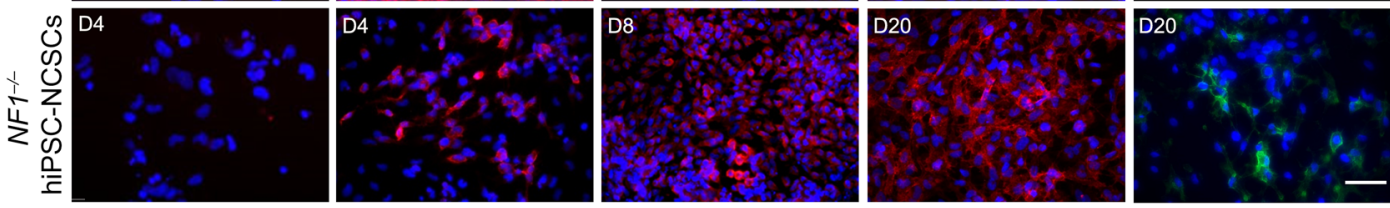

D
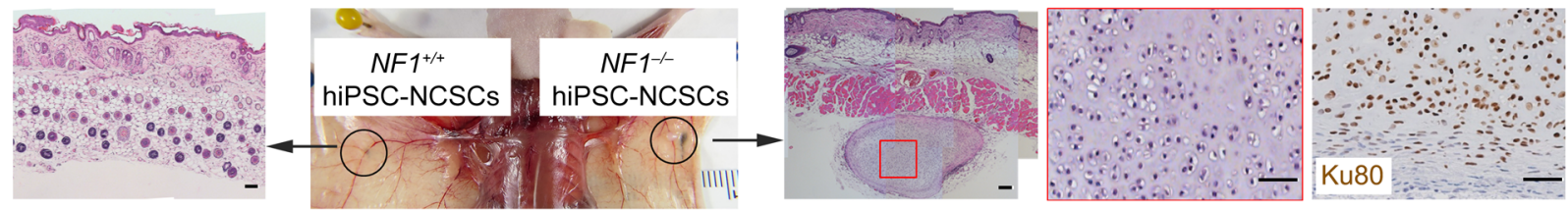

$\mathbf{E}$
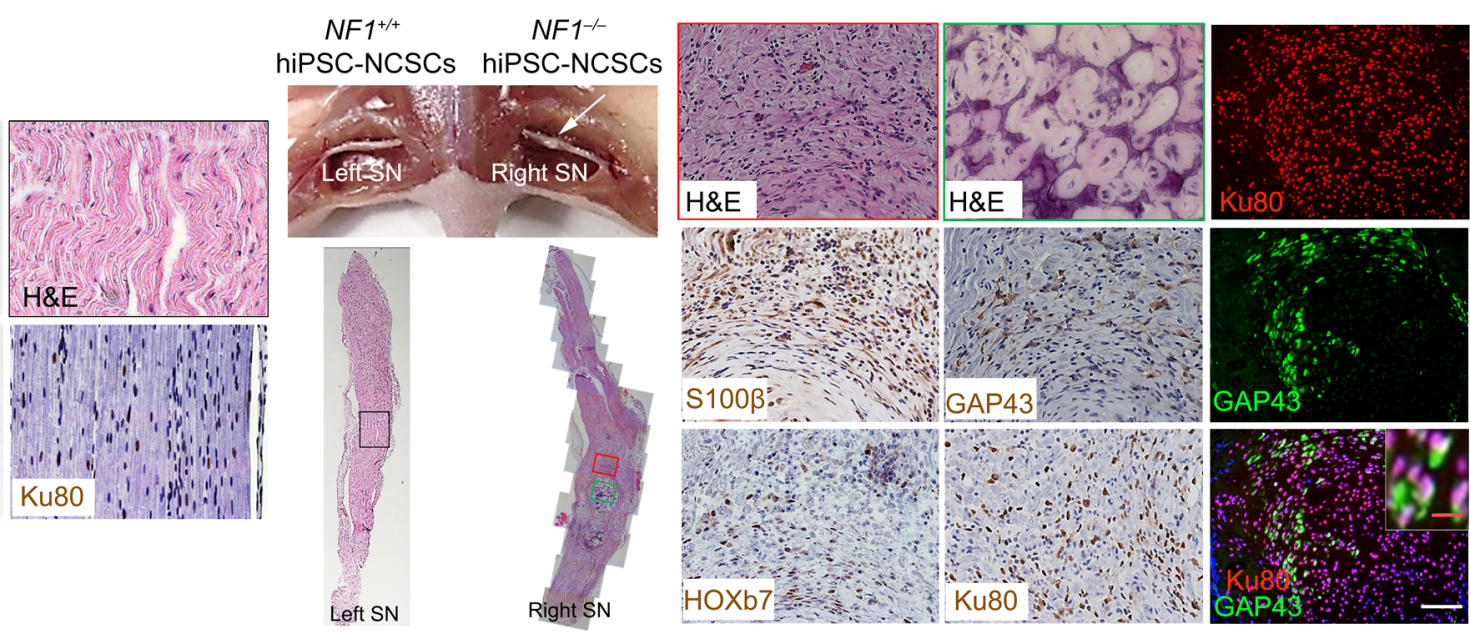

Figure 4. The nerve microenvironment promotes NCSC differentiation into SLCs and the formation of neurofibromas. (A-C) After differentiation, hiPSC-NCSCs were immunonegative for Oct3/4 on day 4, immunopositive for p75 on days 4, 8, and 20, and immunopositive for HNK1 on day 20. (D) hiPSC-NCSCs were subdermally injected into athymic mice. Formation of cartilage derived from injected Ku80+ cells was observed under the skin following $\mathrm{NF}^{-/-}$hiPSC-NCSC implantation, but not in the left sciatic nerve after $N F 7^{+/+}$hiPSC-NCSC implantation. $n=3$. (E) hiPSC-NCSCs were injected into the sciatic nerves of athymic mice. Formation of cartilage and tumors with neurofibroma histological and molecular characteristics was observed in the right sciatic nerves following implantation of NF1/- hiPSC-NCSCs. Colocalization of Ku80 and GAP43 was observed. The left sciatic nerve injected with $\mathrm{NF}^{+/ /+}$ hiPSC-NCSCs was immunopositive for Ku80 but still well-organized, without histological features of neurofibroma. $n=3$. White arrow points to tumor in the right sciatic nerve. Scale bars: $50 \mu \mathrm{m}$ and $10 \mu \mathrm{m}$ (inset in E).

Importantly, when Sox10-CreERT2 expression was induced with tamoxifen in adult Sox10-CreERT2 $\mathrm{Nf1^{ \text {t/l } }} \mathrm{R} 26$-LacZ mice older than 1 month of age, multiple nodules formed that protruded from inside the skin (Supplemental Figure 7B), typical of human sessile cNFs (51). These tumors were more hypercellular relative to the adjacent healthy skin (Figure 8, A and B). The massive infiltration of LacZ ${ }^{+}$cells (Figure 8A) expressing SOX10 (Figure 8A) confirmed that these neoplastic cells were of SOX10 lineage. The tumor area was also immunopositive for SLC markers (S100 $\beta$, GAP43, SOX10) and contained $\mathrm{Iba}^{+}$macrophages and numerous mast cells (Figure
8A), which was distinct from the adjacent healthy skin (Figure 8B), similar to their human counterparts (Figure 8C).

The MEK inhibitor selumetinib was recently approved by the FDA for treatment of pNFs. To provide proof-of-principle support for the use of this novel genetically engineered mouse cNF model for future preclinical drug testing, we topically treated cNFs devel-

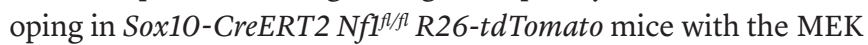
inhibitor PD0325901 (PD901). A small cohort of mice ( $n=3$ per group) were treated thrice daily for 1 week, but then became weak and sick, requiring euthanasia. Analysis of the tumors before and 
A

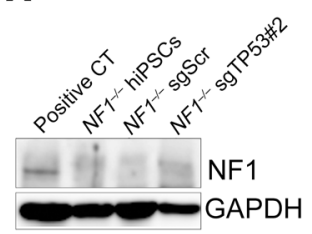

B

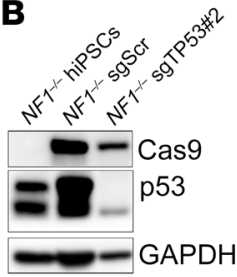

C

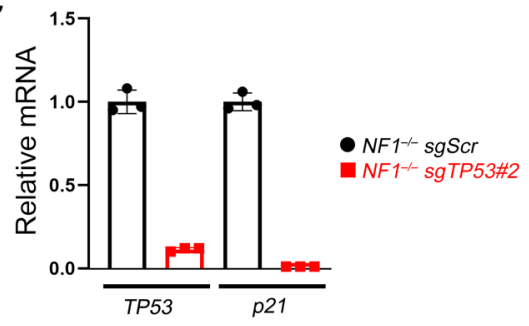

D

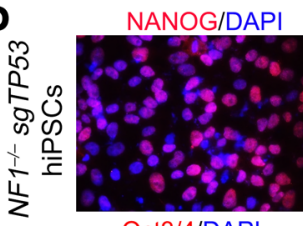

Oct3/4/DAPI

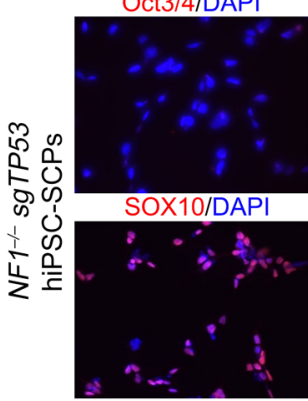

SOX2/DAPI

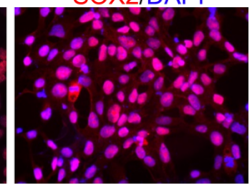

AP2a/DAPI

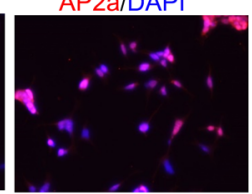

Nestin/DAPI

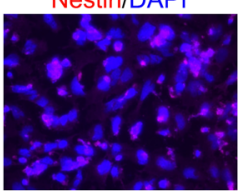

Oct3/4/DAPI

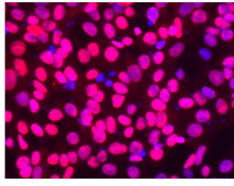

p75/DAPI

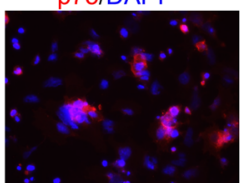

Ki67/DAPI

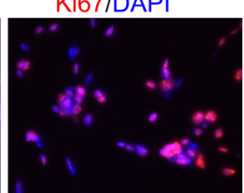

SOX10/DAP

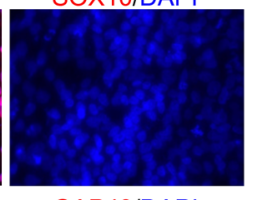

GAP43/DAPI

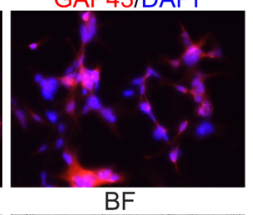

BF

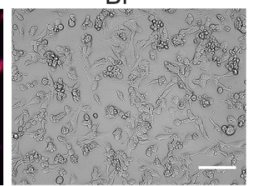

E

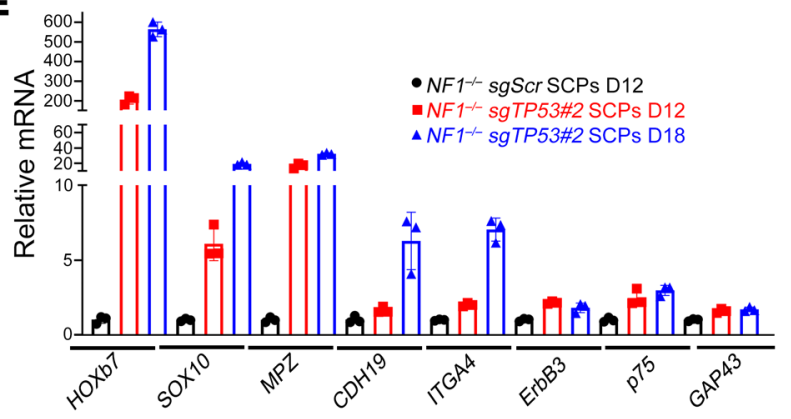

$\mathbf{F}$

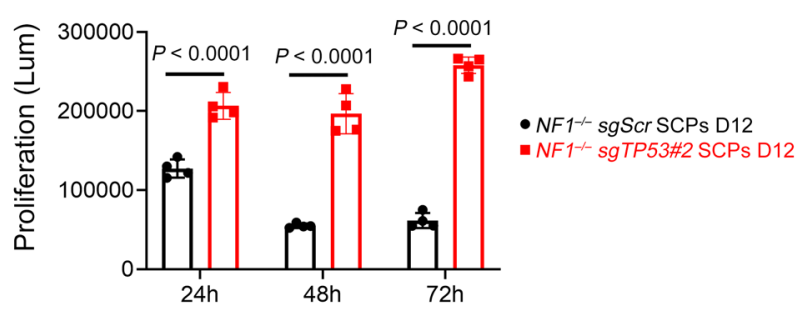

G

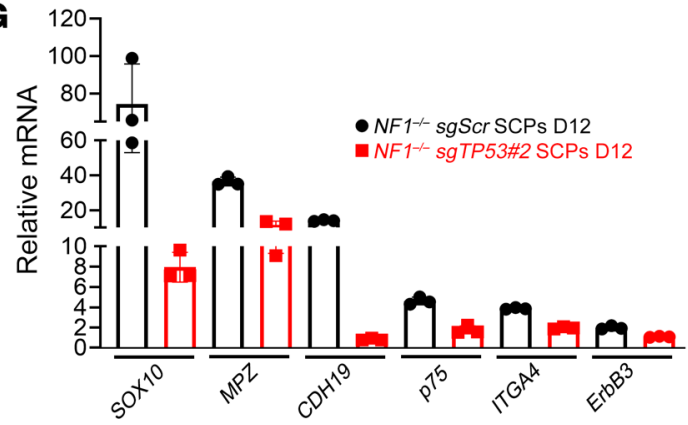

H

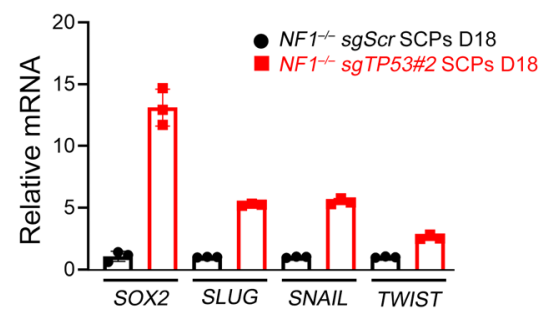

Figure 5. Differentiation into SCPs of hiPSCs with loss of NF1 and TP53. (A and B) TP53 loss was genetically engineered using CRISPR/Cas9 in NF1 hiPSCs. Expression of neurofibromin, Cas9, and TP53 was measured by Western blotting. GAPDH was used as an internal loading control. (C) qPCR was

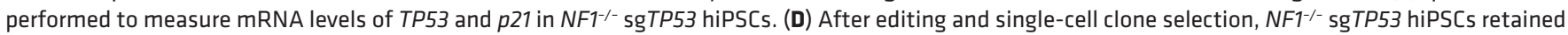
their pluripotency, as verified by the expression of pluripotent markers (NANOG, SOX2, and Oct3/4). However, the SCP marker SOX10 was negative. Differentiation of $\mathrm{NFT}^{-1-}$ sgTP53 hiPSCs into SCPs was confirmed by fluorescence staining using SCP markers (SOX10, AP2a, p75, GAP43, and nestin). Scale bar: $50 \mu \mathrm{m}$. (E) mRNA levels of the indicated SCP markers were measured. (F) Cell proliferation was compared between NF1 ${ }^{-/-}$sgScr hiPSC-SCPs and NF1/sgTP53 hiPSC-SCPs using the CellTiter-Glo assay. Comparisons between groups were performed by 2-way ANOVA. Lum, luminescence. (G and H) mRNA

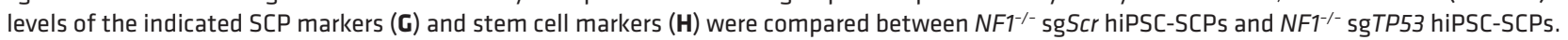

after treatment with PD901 demonstrated that MEK inhibition decreased ERK activity within the cNF (Figure 8, D and E). Since these mice also harbor the R26-tdTomato red reporter, we monitored the response of Sox10-Cre ${ }^{+}$cells to MEK inhibitor treatment using tdTomato red signal. Consistent with reduced p-ERK immunostaining, we found a reduction in $\mathrm{SOX}^{+} \mathrm{O}^{+}$(Figure $8 \mathrm{~F}$ ) and tdTomato red $^{+}$(Figure $8 \mathrm{G}$ ) cells in the treated group compared with the before-treatment or vehicle-treated groups. This proof-of-principle experiment suggests that this murine $\mathrm{cNF}$ model may serve as a tractable platform for future preclinical drug studies.

\section{Discussion}

cNFs develop in nearly all individuals with NF1, can be disfiguring, and greatly affect quality of life. However, little is known about the mechanisms mediating their initiation and progression, and there are no effective medical treatments available. One of the barriers to the identification of promising therapies for $\mathrm{cNFs}$ has been the lack of suitable preclinical platforms. The studies presented here address this pressing need through the generation of robust hiPSCderived neurofibroma and $\mathrm{CNF}$ mouse models. Using these systems, we discovered that NF1 loss impaired Schwann cell lineage 

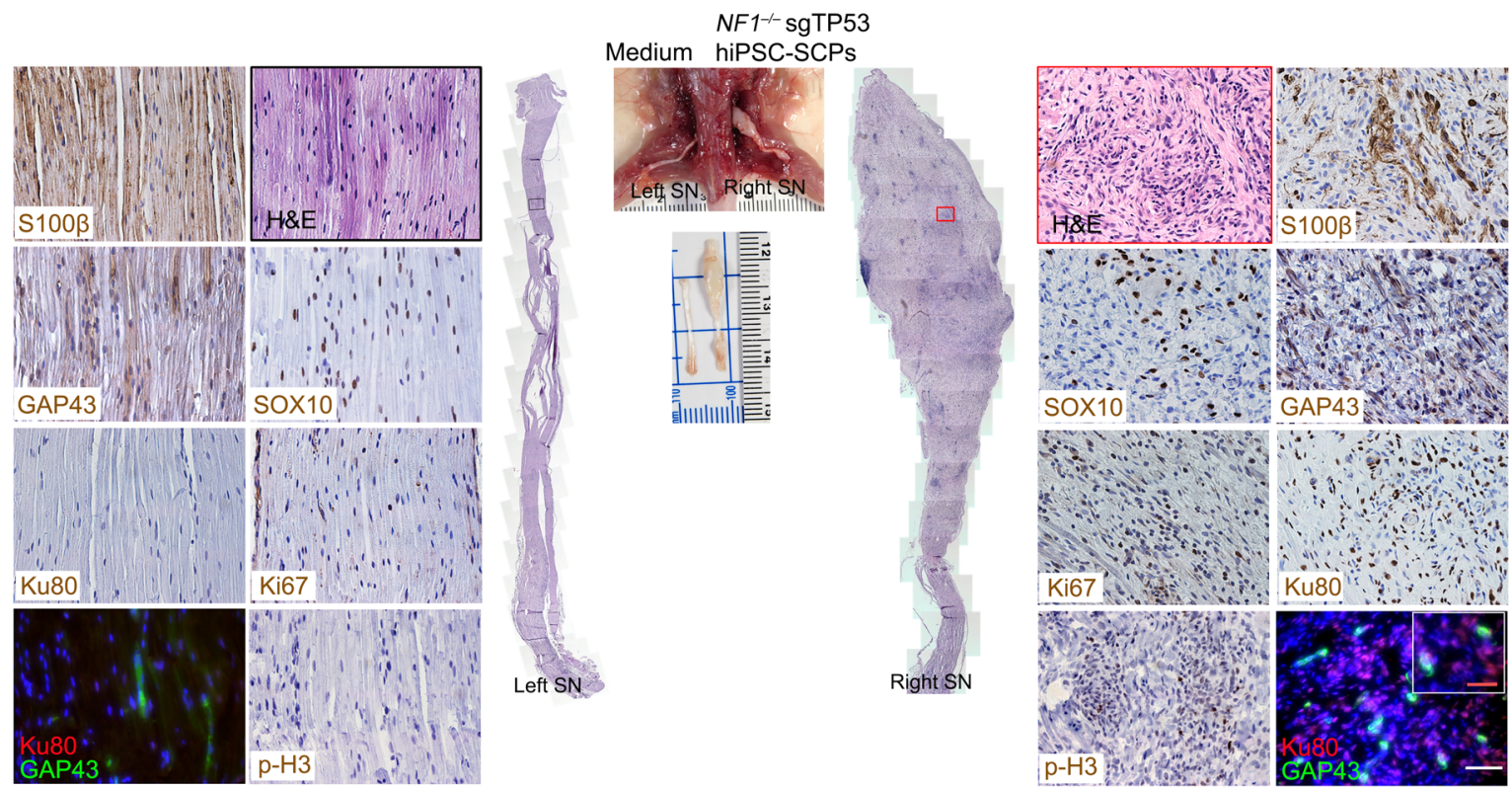

Figure 6. Loss of NF1 and TP53 in hiPSC-SCPs drives MPNST development. After implantation of $\mathrm{NF}^{-1-}$ sgTP53 hiPSC-SCPs into the right sciatic nerve, MPNSTs were observed and characterized by H\&E staining and expression of S100 $\beta$, SOX10, GAP43, Ki67, Ku80, and p-H3. The inset shows the colocalization of $\mathrm{GAP}_{4} 3^{+}$and Ku80+ cells. The left sciatic nerve injected with medium served as a control. $n=3$. Scale bars: $50 \mu \mathrm{m}$ and $25 \mu \mathrm{m}$ (inset).

differentiation by maintaining a more stem-like state, which likely facilitated tumorigenesis. Moreover, the use of a humanized model further underscored the critical importance of a permissive nerve microenvironment to human neurofibroma development. Taken together, the generation and authentication of these neurofibroma models establishes tractable platforms for future preclinical therapeutic discovery and testing.

$S O X 10^{+}$stem cells contain the cells of origin for $c N F s$ and $p N F s$. Prior studies have established the importance of stem cells in the initiation and maintenance of a diverse number of blood and solid cancers $(52,53)$. Although stem cells constitute a small population, the acquisition of cancer-causing mutations favors a proliferative stage that facilitates tumor formation. Using hiPSC-differentiated SCPs and mouse E13.5 DNCSs (containing neurofibroma cells of origin), we discovered that NF1 loss impaired Schwann cell lineage differentiation by arresting cells in a more stem-like state, thus expanding the population of potential cells of origin for neurofibroma.

Using lineage tracing, we found that SOX10 was highly expressed in the DRG and sciatic nerve (Supplemental Figure 5, A and B), where the incidence of pNFs is highest. Consistent with this finding, neurofibroma-generating $\mathrm{NF}^{-/}$hiPSC-SCPs (Figure 1) and humanized neurofibroma tissue (Figure 3A) highly expressed SOX10 (54). Since we have previously shown that HOXb7 labels the cells of origin for neurofibromas in mice (6) and HOXb7 colocalizes with SOX10 expression, we hypothesized that SOX10expressing cells contain the tumor-initiating cells that give rise to neurofibromas. SOX10 is a transcription factor first expressed at E9.5 (55) and maintained throughout Schwann cell lineage differentiation (56), where it regulates the expression of Schwann cell lineage genes, including Oct6 (57), Krox2O (58), and ErbB3 (59). Although SOX10 is not required for initial neural crest specification $(59,60)$, apoptosis of undifferentiated NCSCs and the failure of glial cell generation in the peripheral nerves of SOX1O-mutant mice suggest a critical role for SOX10 in the maintenance of NCSC survival and in glial fate determination $(61,62)$. The high percentage of cNF and pNF co-occurrence in SOX10-CreERT2 NfIt//l mice further underscores the importance of spatiotemporal $\mathrm{NfI}$ loss in SOX10-expressing Schwann cell lineages for the formation of both $\mathrm{cNF}$ and pNF tumors. Importantly, we did not observe optic glioma (or other brain tumor) development in these mice (Supplemental Figure 8), as is seen in 15\%-20\% of children with NF1 (63), since the cells of origin of these tumors are not $\mathrm{SOX}^{+} 0^{+}$cells (64).

Humanized neurofibroma models for personalized medicine. Histologically, human neurofibromas harbor both neoplastic S100 $\beta^{+}$ Schwann cells $\left(\mathrm{NF1}^{-/-}\right)$and non-neoplastic elements $\left(\mathrm{NF1}^{+/-}\right.$cells $)$ in the tumor microenvironment $\left(\mathrm{CD} 34^{+}\right.$fibroblasts, mast cells, and macrophages and nerve fibers) (22). These neuropathological features were faithfully recapitulated in the humanized neurofibroma model system and in the new murine $\mathrm{CNF}$ strain presented in this study. Another notable feature of human neurofibromas is the formation of Meissner-like corpuscles (65), which we observed in the humanized patient-based hiPSC pNF model (Figure 3A) and which, to our knowledge, has not been previously observed in prior genetically engineered mouse models.

Relevant to human clinical trials targeting the neurofibroma tumor microenvironment (e.g., imatinib) (12) or the neoplastic Schwann cells (e.g., MEK inhibitors) (66), tumor responses can vary greatly among patients. These observations argue for the development of robust, authenticated preclinical systems to discover and evaluate potential personalized therapies. Unfortunately, primary tumor cells isolated from neurofibromas of patients with NF1 have limited proliferative capacity and do not form tumors when explanted into mice. Leveraging our prior experience in defining the cells of origin for cNFs $(6)$ and pNFs $(4,9,50)$ in 
A

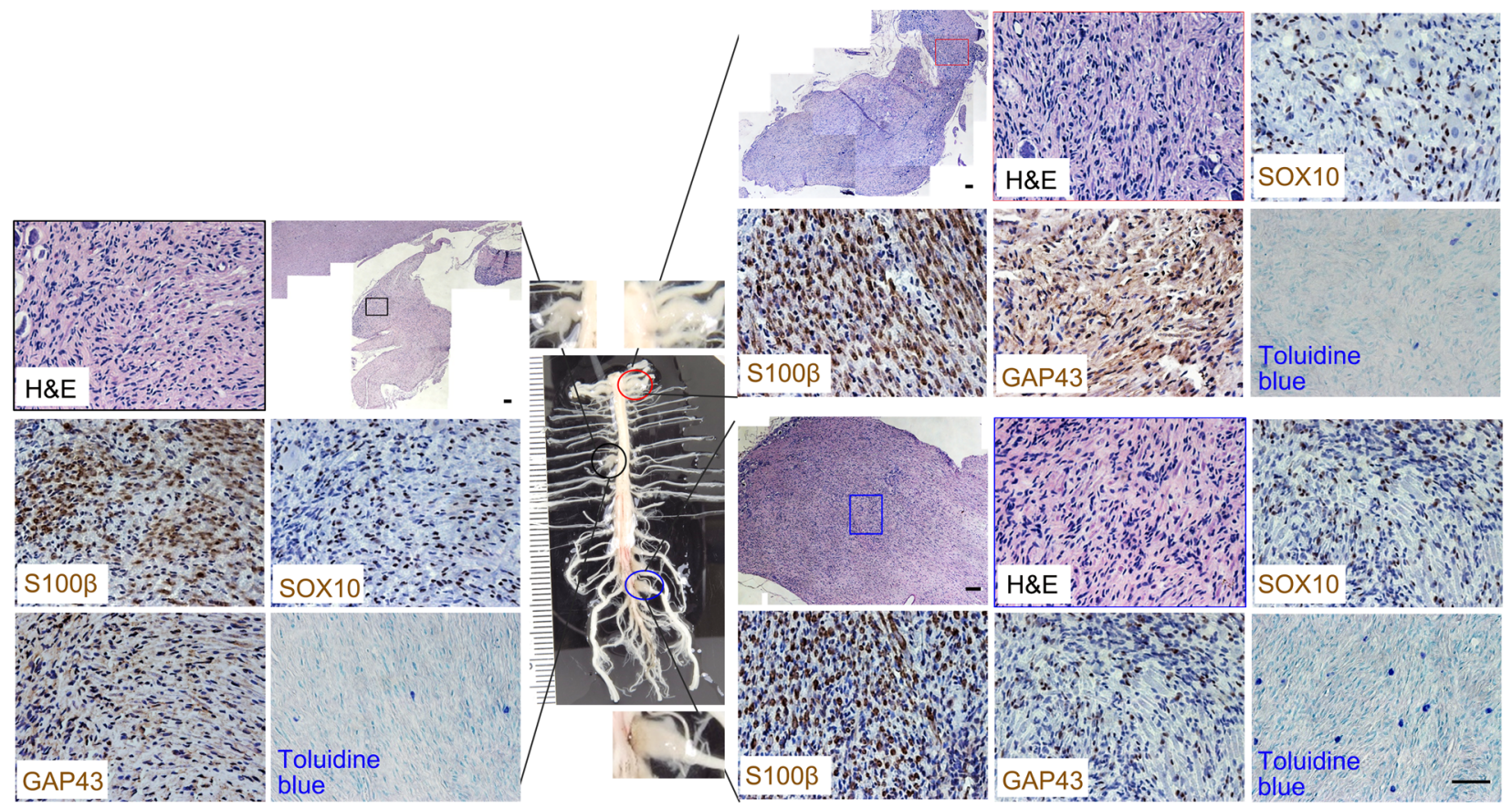

B

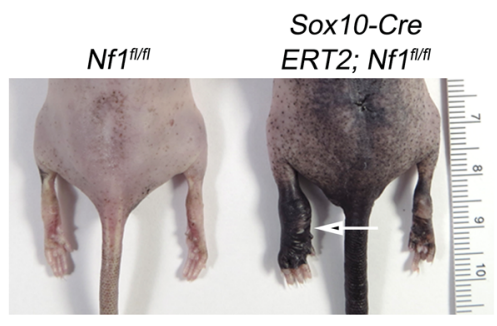

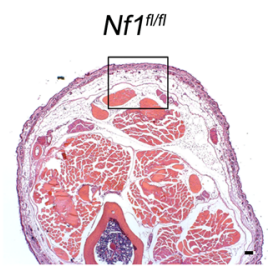

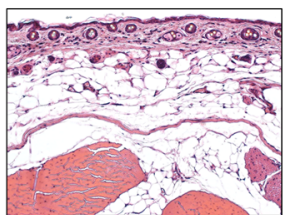

Sox10-CreERT2; Nf1 $1^{1 / t / 1}$

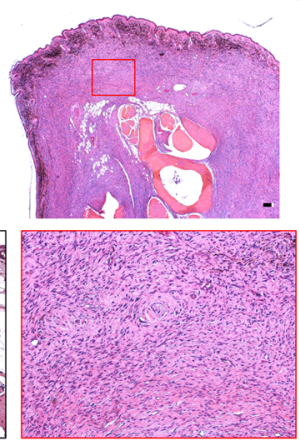

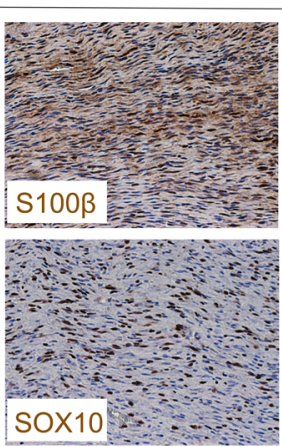

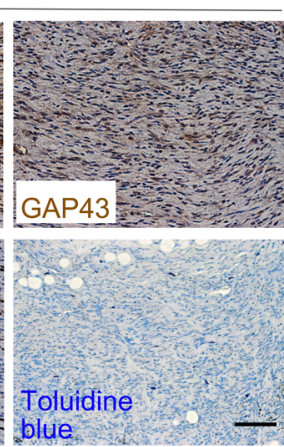

Figure 7. S0X10-expressing cells contain proliferating tumorigenic cells for pNF. (A) Sox10-CreERT2 Nffl/fl mice treated with tamoxifen demonstrated neurofibroma formation, characterized by abnormally enlarged DRGs, as well as hypercellular and disorganized DRGs. The pNF was positive for S100 $\beta$, GAP43, and SOX10 expression, with infiltration of mast cells. (B) A representative Sox10-CreERT2 Nf1 fl/fl mouse treated with tamoxifen developed classic giant, diffuse pNFs (white arrow) with hyperpigmentation and thickening of the skin, which was positive for S100 $\beta$, GAP43, and SOX10 expression, with mast cell infiltration. $n=43$. Scale bars: $50 \mu \mathrm{m}$.

mice, we successfully used hiPSCs to generate humanized models of NF1-associated neurofibromas. The availability of this humanized platform now permits an examination of potential factors that influence neurofibroma formation. One of these potential risk factors is the germline NF1 gene mutation. In this regard, patients with specific germline NF1 gene mutations (Arg1809 missense mutations, c.2970_2972 deletion) fail to develop cutaneous or dermal neurofibromas $(67,68)$. Future studies using these NF1-mutant hiPSC lines will permit mechanistic studies aimed at defining how NF1 gene mutations differentially promote tumor formation (20).

Taken together, we used converging human and mouse modeling methods to identify the common cells of origin for different types of neurofibroma and developed complementary models representing the full range of benign and malignant PNSTs. The use of these models as platforms to understand the molecular and cellular evolution of neurofibroma development, as well as for test- ing potential promising therapeutic compounds, will be of utmost importance in reducing the morbidity and mortality associated with these tumors in patients with NF1.

\section{Methods}

Mice. Athymic nude mice were purchased from Charles River Laboratories (stock no. 490). The $\mathrm{Nf}^{f / / f l}$ mice have been previously described (1). For inducible conditional Nf1 ablation, we used Sox1O-CreERT2transgenic mice, a tamoxifen-inducible Cre mouse line, from The Jackson Laboratory (stock no. 027651). For noninducible conditional $\mathrm{Nf1}$ ablation, we used Sox1O-Cre-transgenic mice from The Jackson Laboratory (stock no. 025807). ROSA26-LacZ reporter mice were obtained from The Jackson Laboratory.

Isogenic hiPSC generation and culturing. Commercially available BJFF.6 healthy human fibroblasts were reprogrammed into iPSCs $\left(\mathrm{NF}^{+/+}\right)$and were subsequently CRISPR/Cas9 engineered at the 
A

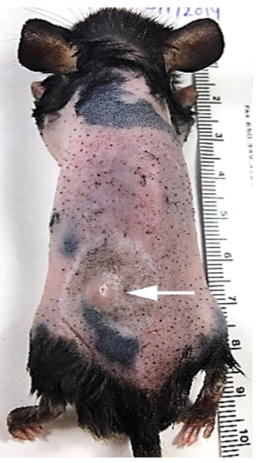

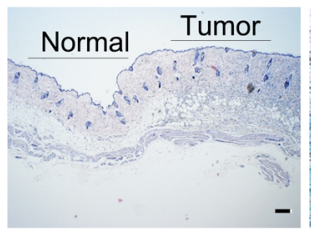
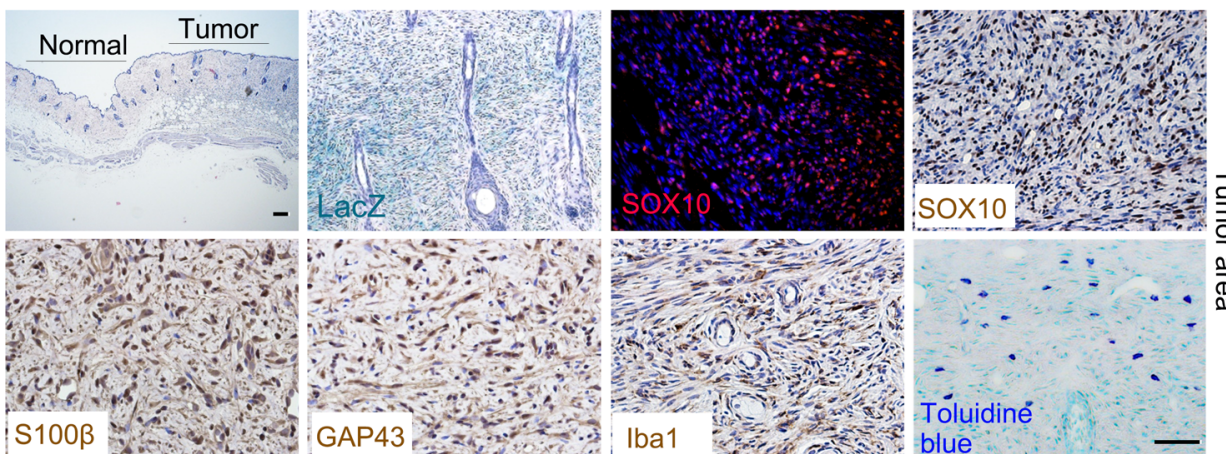

B
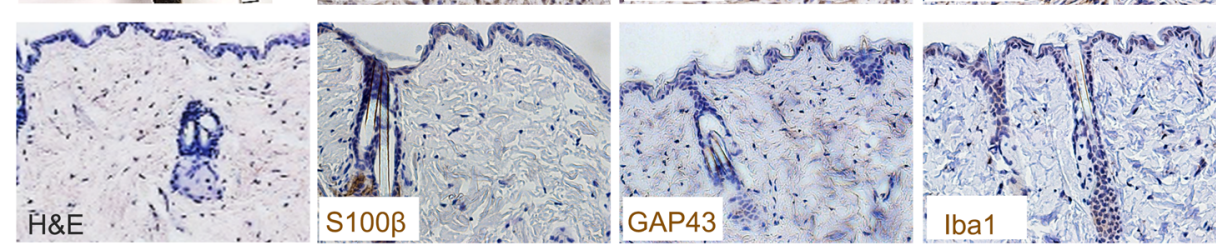

Toluidine

blue
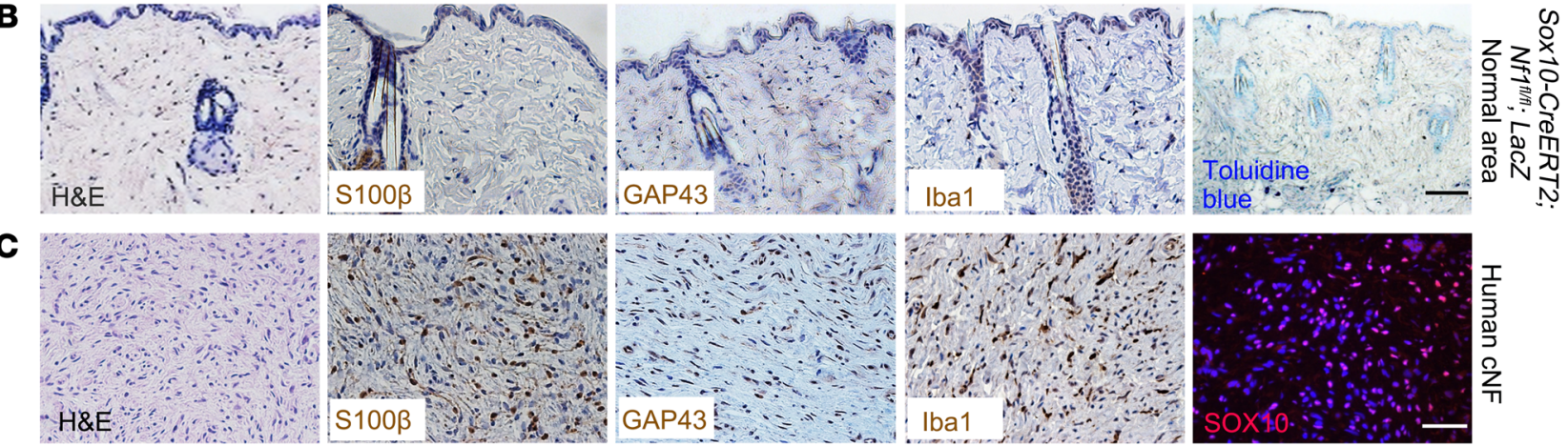

D
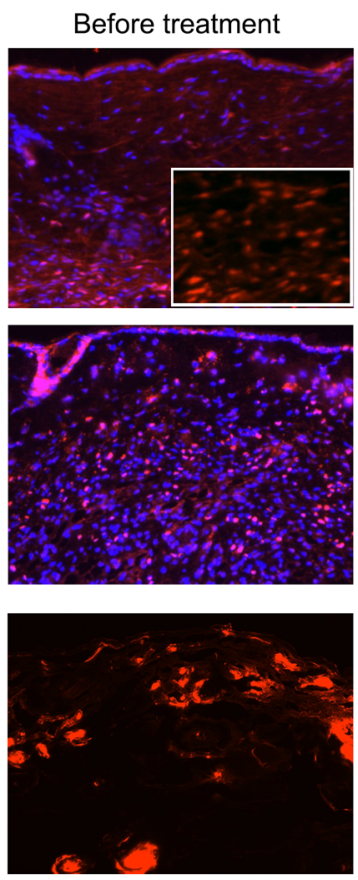

Vehicle treatment
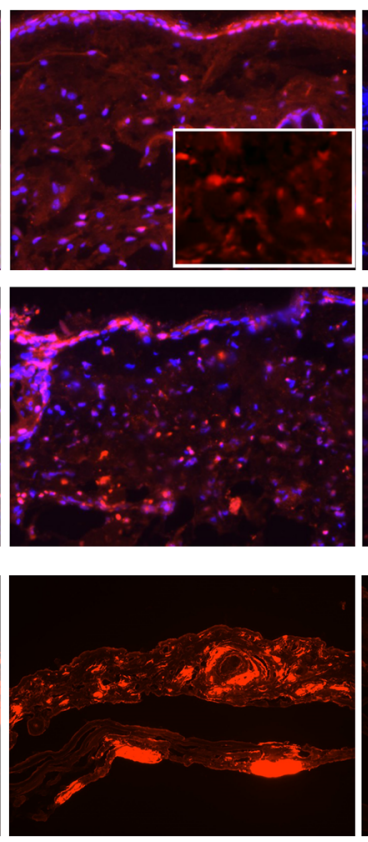

PD901 treatment
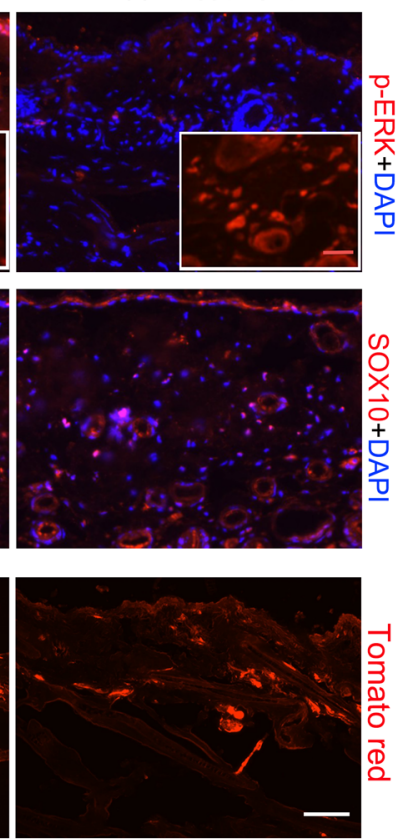

E

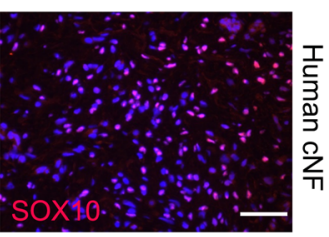

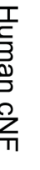

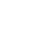


Washington University Genome Engineering and iPSC Core (GEiC) to harbor a heterozygous $\left(\mathrm{NF}^{+/-}\right)$or homozygous $\left(\mathrm{NF1}^{-/}\right) \mathrm{NF1}$ mutation found in patients with NF1: $6619 \mathrm{C}>\mathrm{T}$, c.6513T $>$ A, or c.2041C $>\mathrm{T}$ (20). hiPSCs were maintained in feeder-free, Matrigel-coated plates in mTeSR Plus medium (STEMCELL Technologies).

SCP induction. SCP induction was performed using previously described protocols (69) with modifications. Briefly, hiPSCs were dissociated by Accutase (STEMCELL Technologies) and plated onto Matrigel-coated plates at a density of 25,000 cells $/ \mathrm{cm}^{2}$ in $\mathrm{mTeSR}$ Plus medium containing $10 \mu \mathrm{M}$ ROCK inhibitor (STEMCELL Technologies). Differentiation was initiated by switching to SCP differentiation medium (SCP-DM) when the confluence was approximately $70 \%-80 \%$. The SCP-DM contained 50\% DMEM/F12 (Gibco, Thermo Fisher Scientific), 50\% Neurobasal medium (Gibco, Thermo Fisher Scientific), N2 (Gibco, Thermo Fisher Scientific), B27 (Gibco, Thermo Fisher Scientific), GlutaMAX (Corning), nonessential amino acids (Corning), 2 $\beta$-ME (Invitrogen, Thermo Fisher Scientific), $50 \mathrm{ng} / \mathrm{mL}$ neuregulin $\beta-1$ (Peprotech), $3 \mu \mathrm{M}$ CHIR99021 (STEMCELL Technologies), and $10 \mu \mathrm{M}$ SB431542 (MilliporeSigma). The medium was changed every other day. Detailed information on each product is listed in Supplemental Table 2.

Neurosphere assessment. Neurosphere assessments were performed using previously published protocols (19). hiPSC-SCPs were dissociated by Accutase (STEMCELL Technologies) into single cells and suspended in SCP-DM. The cells were plated onto ultra-low 6-well plates (Corning) with $5 \times 10^{4}$ to $1 \times 10^{5}$ in $3 \mathrm{~mL}$ and allowed 6-14 days for neurosphere formation. The images of each neurosphere were captured by camera, and the diameter of each neurosphere was measured in Photoshop. The average diameter of neurospheres from isogenic hiPSC-SCPs was calculated in Excel. The frequency of isogenic hiPSC-SCPs was assessed as the percentage of cells that could undergo self-renewal and form multipotent neurospheres. Neurospheres and medium were transferred into $15 \mathrm{~mL}$ conical tubes and collected by centrifugation at $100 \mathrm{~g}$ for 10 minutes. After removing the supernatant and washing the cells with DPBS without $\mathrm{Ca}^{2+} / \mathrm{Mg}^{2+}$, the neurospheres were digested with $0.25 \%$ Trypsin/EDTA (MilliporeSigma) for 5 minutes in a $37^{\circ} \mathrm{C}$ incubator. The neurospheres were gently triturated to create single-cell suspensions. The number of cells needed to form neurospheres was counted using a hemocytometer (Thermo Fisher Scientific).

NCSC induction. The NCSC induction protocol described in previous publications was optimized (70). Briefly, hiPSCs were dissociated by Accutase and plated on Matrigel-coated plates at a density of 25,000 cells $/ \mathrm{cm}^{2}$ in mTeSR Plus medium containing $10 \mu \mathrm{M}$ ROCK inhibitor. When cells reached $70 \%$ confluence, differentiation was initiated by switching to NCSC differentiation medium (NCSC-DM) composed of DMEM/F12, 2\% BSA (w/v), GlutaMAX, nonessential amino acids, Trace Elements A, Trace Elements B, Trace Elements C (all from Corning), 2 $\beta-\mathrm{ME}$ (Invitrogen, Thermo Fisher Scientific), $10 \mu \mathrm{g} /$ $\mathrm{mL}$ bovine transferrin (Invitrogen, Thermo Fisher Scientific), $50 \mu \mathrm{g} /$ $\mathrm{mL}(+)$-sodium L-ascorbate (MilliporeSigma), $200 \mathrm{ng} / \mathrm{mL}$ LONGR3 IGF-I (MilliporeSigma), $10 \mathrm{ng} / \mathrm{mL}$ human recombinant FGF2 (Thermo Fisher Scientific), $8 \mathrm{ng} / \mathrm{mL}$ neuregulin $\beta-1$ (Peprotech), $3 \mu \mathrm{M}$ CHIR99021 (STEMCELL Technologies), and $10 \mu \mathrm{M}$ SB431542 (MilliporeSigma). The medium was changed every other day. Detailed information for each product is listed in Supplemental Table 2.

Implantation of cells into the sciatic nerves of immunocompromised mice. Implantation of cells into the sciatic nerve was performed as previously described (4). Briefly, nude (Foxn1-mutant) mice were anesthetized ( $4 \mu \mathrm{L} / \mathrm{g}$, body weight) by intraperitoneal injection using a mixture of ketamine $(10 \mathrm{mg} / \mathrm{mL})$ and xylazine $(1 \mathrm{mg} / \mathrm{mL})$ solution (provided by the UT Southwestern Animal Resource Center [ARC], Dallas, Texas, USA). Using iris scissors, a skin incision was made above the femur. A pocket was formed, and the sciatic nerve was exposed by displacing the quadriceps muscle. $\mathrm{L}-15$ medium $(50 \mu \mathrm{L})$ containing $1 \times 10^{6}$ viable hiPSC-differentiated SCPs was then deposited into the pocket so that cells were in contact with the injured sciatic nerve. The quadriceps muscles was then closed with 4-0 Vicryl sutures (Ethicon), and the skin was closed with staples. Mice were placed into warm cages to recover from anesthesia.

MEK inhibitor treatment. The MEK inhibitor PD0325901 (PD901) (Selleckchem) was dissolved in 100\% DMSO (MilliporeSigma). Immediately before topical application, this solution was diluted 1:1 with water. A total of $1 \mathrm{mg}$ PD 901 was applied to the backs of Sox10-CreERT2 $N f 1^{f / f l} \mathrm{R} 26$ - $t$ Thomato mouse skin bearing cNFs thrice daily with inhibitor solution (3 mg total) or 50\% DMSO (control). White petrolatum was used to occlude the inhibitor. Tumors were harvested for analysis after 1 week of treatment, when mice began to be weak and sick. Quantification of the tdTomato red $^{+}$cells was performed using ImageJ (NIH).

FACS. $\mathrm{NF1}^{+/+}, \mathrm{NF1}^{+/-}$, and $\mathrm{NF1}^{-/-}$hiPSC-SCPs were dissociated using Accutase and resuspended in FACS buffer (Dulbecco's PBS [DPBS] without $\mathrm{Ca}^{2+} / \mathrm{Mg}^{2+}$ containing $0.5 \mu \mathrm{M}$ EDTA and $1 \% \mathrm{BSA}$ ) to a final concentration of $5 \times 10^{6}$ cells $/ \mathrm{mL}$. Nonspecific staining of cells was blocked with Human BD Fc Block (BD Pharmingen) for 30 minutes on ice. A total of $200 \mu \mathrm{L}$ of each sample was added to duplicate Falcon tubes for staining with allophycocyanin-conjugated (APC-conjugated) isotype control or APC-conjugated p75 antibodies for 1 hour on ice. Each sample was washed twice with FACS buffer and resuspended in $500 \mu \mathrm{L}$ FACS buffer. After propidium iodide (PI) was added, the cell suspension was transferred into the appropriate tubes for FACS. The $\mathrm{PI}^{-} \mathrm{APC}^{+}$cell population was analyzed with FlowJo software.

Histology and immunostaining. For H\&E analysis, tissue specimens were harvested and fixed with $10 \%$ formalin in PBS for 1 day and subsequently embedded in paraffin. Sections ( $5 \mu \mathrm{m}$ thick) were stained with H\&E according to the manufacturer's protocol (StatLab). The antibodies used for immunohistochemistry and immunofluorescence studies are listed in Supplemental Table 3.

$q R T-P C R$ and analysis. Total RNA was extracted using TRIzol Reagent (Life Technologies, Thermo Fisher Scientific), and $1 \mu \mathrm{g}$ RNA was reverse transcribed with an iScript Select cDNA Synthesis Kit (Bio-Rad). The primer sequences are listed in Supplemental Table 4. Real-time quantitative PCR (qRT-PCR) mixtures were prepared with iTaq Universal SYBR Green Supermix (Bio-Rad), and reactions were performed on a CFX connect Real-time System (Bio-Rad). Ct values were normalized to the housekeeping gene Actb.

Plasmid construction. To silence TP53 expression in $\mathrm{NF1}^{-/-}$hiPSCs, annealed sgRNAs targeting TP53 were ligated to a digested CRISPRv2 vector. After transformation of the ligation product into Stbl3-competent cells and screening single clones on ampicillin agar plates, the resulting clone was expanded in Luria-Bertani (LB) broth, and plasmids were extracted using NucleoBond Xtra Midi (Macherey-Nagel). The sgRNA primers used in this study are listed in Supplemental Table 5.

Genomic PCR. Genomic PCR was performed to identify the clones containing CRISPRv2 plasmids with the sgRNA insertion using 2XTaq RED Master Mix (Apex). The primer sequences are listed in Supplemental Table 5. 
Genotyping. To determine the genotypes of the genetically modified mice, genomic DNA was extracted from tails (pups) using $1 \mathrm{M}$ Tris- $\mathrm{HCl}$ (pH 6.8) and incubated for 1 hour followed by $50 \mathrm{mM} \mathrm{NaOH}$ neutralization. The DNA sequences including the target site were amplified with 2XTaq RED Master Mix (Apex). The primer sequences are listed in Supplemental Table 6.

4-Hydroxytamoxifen and tamoxifen treatment. 4-Hydroxytamoxifen (MilliporeSigma) was dissolved in $100 \%$ ETOH at $4 \mathrm{mg} / \mathrm{mL}$. PO pups were injected subcutaneously with $40 \mu \mathrm{g} / \mu \mathrm{L}$ 4-hydroxytamoxifen. For adult mice, tamoxifen (MilliporeSigma) was dissolved in a sunflower oil/ethanol mixture (9:1) at $10 \mathrm{mg} / \mathrm{mL}$. The mice were gavaged with $2 \mathrm{mg} / 200 \mu \mathrm{L}$ tamoxifen for 5 consecutive days. Mice were genotyped before and after injection to confirm the deletion of genes flanked by loxP sites using the $N f 1$ primers listed in Supplemental Table 6 .

Supplemental Table 7 lists the different genotypes of the mice and their phenotypes.

Study approval. The care and use of animals in this study were approved by the IACUC of the University of Texas Southwestern Medical Center.

Statistics. Values for each sample were from at least 3 biologically independent experiments with at least 3 technical replicates. Data are reported as the mean \pm SEM. Comparisons among groups were performed by 1-way ANOVA, 2-way ANOVA, or 2-tailed Student's $t$ test for pair-wise comparisons. $P$ values of 0.05 or less were considered statistically significant.

\section{Author contributions}

LQL, JM, and DHG conceived and designed the study. LQL, JM, and ZC developed the methodology. LQL, JM, ZC, CA, TS, KY, and JP acquired data, provided animals, generated cells and reagents, and provided facilities. LQL and JM analyzed and interpreted the data. LQL, JM, CA, and DHG wrote, reviewed and revised the manuscript. LQL and JM provided administrative, technical, and material support in the form of reporting and organizing data and constructing databases. LQL supervised the study.

\section{Acknowledgments}

We thank the members of the Le and Gutmann laboratories for helpful discussions, Yong Wang for technical assistance, and Renee McKay for reviewing and editing the manuscript. JM is the recipient of the Early Investigator Research Award from the US Department of Defense (W81XWH1910687). CA is funded by a National Cancer Institute (NCI) Research Specialist Award (R50CA233164). LQL holds the Thomas L. Shields, MD Professorship in Dermatology and is supported by the NF1 Research Consortium Fund and the Neurofibromatosis Therapeutic Acceleration Program. DHG holds the Donald O. Schnuck Family Professorship in Neurology. This work was supported by funding from the Giorgio Foundation (to LQL and DHG), the NCI (R01 CA166593 and U54 CA 196519, to LQL), and the NIH (R35 NS097211, to DHG).

Address correspondence to: Lu Q. Le, Department of Dermatology, Simmons Comprehensive Cancer Center, Hamon Center for Regenerative Science and Medicine, UT Southwestern Medical Center, 5323 Harry Hines Blvd., Dallas, Texas 75390, USA. Phone: 214.648.5781; Email: Lu.Le@UTSouthwestern.edu. Or to: David H. Gutmann, Department of Neurology, Washington University School of Medicine in St. Louis, Box 8111, 660 South Euclid Avenue, St. Louis, Missouri 63110, USA. Phone: 314.362.7379; Email: gutmannd@wustl.edu.
1. Zhu Y, Ghosh P, Charnay P, Burns DK, Parada LF. Neurofibromas in NF1: Schwann cell origin and role of tumor environment. Science. 2002;296(5569):920-922.

2. Zheng $\mathrm{H}$, et al. Induction of abnormal proliferation by nonmyelinating schwann cells triggers neurofibroma formation. Cancer Cell. 2008;13(2):117-128.

3. Wu J, et al. Plexiform and dermal neurofibromas and pigmentation are caused by Nf1 loss in desert hedgehog-expressing cells. Cancer Cell. 2008;13(2):105-116.

4. Chen Z, Liu C, Patel AJ, Liao CP, Wang Y, Le LQ. Cells of origin in the embryonic nerve roots for NF1-associated plexiform neurofibroma. Cancer Cell. 2014;26(5):695-706.

5. Mayes DA, et al. Perinatal or adult Nf1 inactivation using tamoxifen-inducible PlpCre each cause neurofibroma formation. Cancer Res. 2011;71(13):4675-4685.

6. Chen Z, et al. Spatiotemporal loss of NF1 in Schwann cell lineage leads to different types of cutaneous neurofibroma susceptible to modification by the hippo pathway. Cancer Discov. 2019;9(1):114-129.

7. Radomska KJ, et al. Cellular origin, tumor progression, and pathogenic mechanisms of cutaneous neurofibromas revealed by mice with $N f 1$ knockout in boundary cap cells. Cancer Discov. 2019;9(1):130-147.
8. Brosseau JP, et al. NF1 heterozygosity fosters de novo tumorigenesis but impairs malignant transformation. Nat Commun. 2018;9(1):5014.

9. Liao CP, et al. Contributions of inflammation and tumor microenvironment to neurofibroma tumorigenesis. JClin Invest. 2018;128(7):2848-2861.

10. Yang FC, et al. Nf1-dependent tumors require a microenvironment containing Nf1+/-and c-kit-dependent bone marrow. Cell. 2008;135(3):437-448.

11. Hirbe AC, Dahiya S, Friedmann-Morvinski D, Verma IM, Clapp DW, Gutmann DH. Spatiallyand temporally-controlled postnatal p53 knockdown cooperates with embryonic Schwann cell precursor Nf1 gene loss to promote malignant peripheral nerve sheath tumor formation. Oncotarget. 2016;7(7):7403-7414.

12. Robertson KA, et al. Imatinib mesylate for plexiform neurofibromas in patients with neurofibromatosis type 1: a phase 2 trial. Lancet Oncol. 2012;13(12):1218-1224.

13. Ferguson MJ, et al. Preclinical evidence for the use of sunitinib malate in the treatment of plexiform neurofibromas. Pediatr Blood Cancer. 2016;63(2):206-213.

14. Gross AM, et al. Selumetinib in children with inoperable plexiform neurofibromas. $\mathrm{NEngl} \mathrm{J}$ Med. 2020;382(15):1430-1442.

15. Jessen KR, Mirsky R. The origin and development of glial cells in peripheral nerves. Nat Rev
Neurosci. 2005;6(9):671-682.

16. Riethmacher D, Sonnenberg-Riethmacher E, Brinkmann V, Yamaai T, Lewin GR, Birchmeier C. Severe neuropathies in mice with targeted mutations in the ErbB3 receptor. Nature. 1997;389(6652):725-730.

17. Furlan A, et al. Multipotent peripheral glial cells generate neuroendocrine cells of the adrenal medulla. Science. 2017;357(6346):eaal3753.

18. Joseph NM, et al. Neural crest stem cells undergo multilineage differentiation in developing peripheral nerves to generate endoneurial fibroblasts in addition to Schwann cells. Development 2004;131(22):5599-5612.

19. Joseph NM, et al. The loss of Nf1 transiently promotes self-renewal but not tumorigenesis by neural crest stem cells. Cancer Cell. 2008;13(2):129-140.

20. Anastasaki C, et al. Human iPSC-derived neurons cerebral organoids establish differential effects of germline NF1 gene mutations. Stem Cell Rep. 2020;14(4):541-550.

21. Schmid RS, et al. Neuregulin 1-erbB2 signaling is required for the establishment of radial glia and their transformation into astrocytes in cerebral cortex. Proc Natl Acad Sci US A. 2003;100(7):4251-4256.

22. Le LQ, Shipman T, Burns DK, Parada LF. Cell of origin and microenvironment contribution for NF1-associated dermal neurofibromas. Cell Stem 
Cell. 2009;4(5):453-463.

23. Bollag G, et al. Loss of NF1 results in activation of the Ras signaling pathway and leads to aberrant growth in haematopoietic cells. Nat Genet. 1996;12(2):144-148.

24. Chen YH, Gianino SM, Gutmann DH. Neurofibromatosis-1 regulation of neural stem cell proliferation and multilineage differentiation operates through distinct RAS effector pathways. Genes Dev. 2015;29(16):1677-1682.

25. Hegedus B, et al. Neurofibromatosis-1 regulates neuronal and glial cell differentiation from neuroglial progenitors in vivo by both CAMP- and Ras-dependent mechanisms. Cell Stem Cell. 2007;1(4):443-457.

26. Iwashita T, Kruger GM, Pardal R, Kiel MJ, Morrison SJ. Hirschsprung disease is linked to defects in neural crest stem cell function. Science. 2003;301(5635):972-976.

27. Schubert J, Brabletz T. p53 Spreads out further: suppression of EMT and stemness by activating miR200c expression. Cell Res. 2011;21(5):705-707.

28. Xue JY, et al. Rapid non-uniform adaptation to conformation-specific KRAS(G12C) inhibition. Nature. 2020;577(7790):421-425.

29. Johannessen CM, Reczek EE, James MF, Brems $\mathrm{H}$, Legius E, Cichowski K. The NF1 tumor suppressor critically regulates TSC2 and mTOR. Proc Natl Acad Sci U S A. 2005;102(24):8573-8578.

30. Liao CP, Pradhan S, Chen Z, Patel AJ, Booker RC, Le LQ. The role of nerve microenvironment for neurofibroma development. Oncotarget. 2016;7(38):61500-61508.

31. Menendez L, Yatskievych TA, Antin PB, Dalton S. Wnt signaling and a Smad pathway blockade direct the differentiation of human pluripotent stem cells to multipotent neural crest cells. Proc Natl Acad Sci U S A. 2011;108(48):19240-19245.

32. McMahon JA, Takada S, Zimmerman LB, Fan CM, Harland RM, McMahon AP. Noggin-mediated antagonism of BMP signaling is required for growth and patterning of the neural tube and somite. Genes Dev. 1998;12(10):1438-1452.

33. Hackland JOS, et al. Top-down inhibition of BMP signaling enables robust induction of hPSCs into neural crest in fully defined, xeno-free conditions. Stem Cell Reports. 2017;9(4):1043-1052.

34. Mica Y, Lee G, Chambers SM, Tomishima MJ, Studer L. Modeling neural crest induction, melanocyte specification, and disease-related pigmentation defects in hESCs and patient-specific iPSCs. Cell Rep. 2013;3(4):1140-1152.

35. Carrio M, et al. Reprogramming captures the genetic and tumorigenic properties of neurofibromatosis type 1 plexiform neurofibromas. Stem Cell Rep. 2019;12(2):411-426.

36. Cichowski K, et al. Mouse models of tumor development in neurofibromatosis type 1. Science. 1999;286(5447):2172-2176.

37. Endo M, et al. Prognostic significance of p14ARF, p15INK4b, and p16INK4a inactivation in malignant peripheral nerve sheath tumors. Clin Cancer Res. 2011;17(11):3771-3782.

38. Gregorian C, et al. PTEN dosage is essential for neurofibroma development and malignant transformation. Proc Natl Acad Sci U S A.
2009;106(46):19479-19484.

39. Huijbregts RPH, Roth KA, Schmidt RE, Carroll SL. Hypertrophic neuropathies and malignant peripheral nerve sheath tumors in transgenic mice overexpressing glial growth factor $\beta 3$ in myelinating Schwann cells. J Neurosci. 2003;23(19):7269-7280.

40. Joseph NM, et al. The loss of Nf1 transiently promotes self-renewal but not tumorigenesis by neural crest stem cells. Cancer Cell. 2008;13(2):129-140.

41. Keng VW, et al. PTEN and NF1 inactivation in Schwann cells produces a severe phenotype in the peripheral nervous system that promotes the development and malignant progression of peripheral nerve sheath tumors. Cancer Res. 2012;72(13):3405-3413.

42. Ling BC, et al. Role for the epidermal growth factor receptor in neurofibromatosis-related peripheral nerve tumorigenesis. Cancer Cell. 2005;7(1):65-75.

43. Perrone F, et al. PDGFRA, PDGFRB, EGFR, and downstream signaling activation in malignant peripheral nerve sheath tumor. Neuro Oncol. 2009;11(6):725-736.

44. Perry A, et al. Differential NF1, p16, and EGFR patterns by interphase cytogenetics (FISH) in malignant peripheral nerve sheath tumor (MPNST) and morphologically similar spindle cell neoplasms. J Neuropathol Exp Neurol. 2002;61(8):702-709.

45. Torres KE, et al. Activated MET is a molecular prognosticator and potential therapeutic target for malignant peripheral nerve sheath tumors. Clin Cancer Res. 2011;17(12):3943-3955.

46. Vogel KS, Klesse LJ, Velasco-Miguel S, Meyers K, Rushing EJ, Parada LF. Mouse tumor model for neurofibromatosis type 1. Science. 1999;286(5447):2176-2179.

47. Lee $\mathrm{W}$, et al. PRC2 is recurrently inactivated through EED or SUZ12 loss in malignant peripheral nerve sheath tumors. Nat Genet. 2014;46(11):1227-1232.

48. Miller SJ, et al. Large-scale molecular comparison of human schwann cells to malignant peripheral nerve sheath tumor cell lines and tissues. Cancer Res. 2006;66(5):2584-2591.

49. Zhu Y, et al. Ablation of NF1 function in neurons induces abnormal development of cerebral cortex and reactive gliosis in the brain. Genes Dev. 2001;15(7):859-876.

50. Le LQ, Liu C, Shipman T, Chen Z, Suter U, Parada LF. Susceptible stages in Schwann cells for NF1-associated plexiform neurofibroma development. Cancer Res. 2011;71(13):4686-4695.

51. Chamseddin BH, Hernandez L, Solorzano D, Vega J, Le LQ. Robust surgical approach for cutaneous neurofibroma in neurofibromatosis type 1 . JCI Insight. 2019;5:128881.

52. Eppert K, et al. Stem cell gene expression programs influence clinical outcome in human leukemia. Nat Med. 2011;17(9):1086-1093.

53. Ricci-Vitiani L, et al. Identification and expansion of human colon-cancer-initiating cells. Nature. 2007;445(7123):111-115.

54. Antonescu CR SB, Woodruff JM. AFIP Atlas of
Tumor Pathology. ARP Press; 2013

55. Pusch C, et al. The SOX10/Sox10 gene from human and mouse: sequence, expression, and transactivation by the encoded HMG domain transcription factor. Hum Genet. 1998;103(2):115-123.

56. Jessen KR, Mirsky R. Schwann cell precursors; multipotent glial cells in embryonic nerves. Front Mol Neurosci. 2019;12:69.

57. Finzsch M, et al. Sox10 is required for Schwann cell identity and progression beyond the immature Schwann cell stage. J Cell Biol. 2010;189(4):701-712.

58. Schreiner S, et al. Hypomorphic Sox10 alleles reveal novel protein functions and unravel developmental differences in glial lineages. Development. 2007;134(18):3271-3281.

59. Britsch S, et al. The transcription factor Sox10 is a key regulator of peripheral glial development. Genes Dev. 2001;15(1):66-78.

60. Southard-Smith EM, Kos L, Pavan WJ. Sox10 mutation disrupts neural crest development in Dom Hirschsprung mouse model. Nat Genet. 1998;18(1):60-64.

61. Paratore C, Goerich DE, Suter U, Wegner M, Sommer L. Survival and glial fate acquisition of neural crest cells are regulated by an interplay between the transcription factor Sox10 and extrinsic combinatorial signaling. Development. 2001;128(20):3949-3961.

62. Sonnenberg-Riethmacher E, Miehe M, Stolt CC, Goerich DE, Wegner M, Riethmacher D. Development and degeneration of dorsal root ganglia in the absence of the HMG-domain transcription factor Sox10. Mech Dev. 2001;109(2):253-265.

63. Campen CJ, Gutmann DH. Optic pathway gliomas in neurofibromatosis type 1.J Child Neurol. 2018;33(1):73-81.

64. Lee DY, Gianino SM, Gutmann DH. Innate neural stem cell heterogeneity determines the patterning of glioma formation in children. Cancer Cell. 2012;22(1):131-138.

65. Ortonne N, et al. Cutaneous neurofibromas: current clinical and pathologic issues. Neurology. 2018;91(2 suppl 1):S5-S13.

66. Dombi E, et al. Activity of selumetinib in neurofibromatosis type 1-related plexiform neurofibromas. N Engl J Med. 2016;375(26):2550-2560.

67. Rojnueangnit $\mathrm{K}$, et al. High incidence of noonan syndrome features including short stature and pulmonic stenosis in patients carrying NF1 missense mutations affecting p.Arg1809: genotype-phenotype correlation. Hum Mutat. 2015;36(11):1052-1063.

68. Koczkowska M, et al. Expanding the clinical phenotype of individuals with a 3-bp in-frame deletion of the NF1 gene (c.2970 2972del): an update of genotype-phenotype correlation. Genet Med. 2019;21(4):867-876.

69. Kim HS, et al. Schwann cell precursors from human pluripotent stem cells as a potential therapeutic target for myelin repair. Stem Cell Reports. 2017;8(6):1714-1726.

70. Menendez L, et al. Directed differentiation of human pluripotent cells to neural crest stem cells. Nat Protoc. 2013;8(1):203-212. 